\title{
Petrology, Geochronology and Geochemistry of Late Triassic Alkaline Rocks of the Bailinchuan District in Liaodong Peninsula, Northeast China
}

\author{
Xihui Cheng ${ }^{1,2, *}$, Jiuhua $\mathrm{Xu}^{2}{ }^{2}$, Hao Wei ${ }^{3}$, Fuquan Yang ${ }^{1}$, Hui Zhang ${ }^{2}$ and Guorui Zhang ${ }^{3}$ \\ 1 MNR Key Laboratory of Metallogeny and Mineral Assessment, Institute of Mineral Resources, \\ Chinese Academy of Geological Sciences, Beijing 100037, China; yangfuquan66@sina.com \\ 2 School of Civil and Resource Engineering, University of Science and Technology Beijing, \\ Beijing 100083, China; jiuhuaxu@ces.ustb.edu.cn (J.X.); huizhangustb@126.com (H.Z.) \\ 3 Geological Testing Center, Hebei GEO University, Shijiazhuang 050031, China; ronghaiwei@163.com (H.W.); \\ zgrsdyney@163.com (G.Z.) \\ * Correspondence: cheng_xihui@163.com
}

Received: 18 August 2018; Accepted: 8 November 2018; Published: 13 November 2018

\begin{abstract}
The Bailinchuan alkaline syenite (BAS) is located in the easternmost part of the Triassic alkaline magmatic belt along the northern North China Craton (NCC). Based on a detailed study of the zircon $\mathrm{U}-\mathrm{Pb}$ age, petrological, and geochemical data of the complex, the characteristics of the magmas system, petrogenesis and the nature of mantle source provide new constraints on the origin and tectonic setting of the Triassic alkaline belt. The BAS is composed of alkaline syenite and/or aegirine-nepheline syenite, with zircon $\mathrm{U}-\mathrm{Pb}$ age of 226-229 Ma. Aegirine, Na-rich augite, biotite, orthoclase, and nepheline are the major minerals. Most of the zircons selected for the analysis show fine-scale to weak oscillatory growth zoning in CL images, suggesting a magmatic origin. Mineralogy, petrology and geochemical studies show that the parental magma of the $\mathrm{BAS}$ is $\mathrm{SiO}_{2}$-undersaturated, potassic, and is characterized by high contents of $\mathrm{CaO}, \mathrm{Fe}_{2} \mathrm{O}_{3}, \mathrm{~K}_{2} \mathrm{O}, \mathrm{Na}_{2} \mathrm{O}$. The BAS originated from a phlogopite-rich, enriched lithospheric mantle source in a garnet-stable area. The occurrence of the BAS, together with many other alkaline complexes of similar ages (235-209 Ma) in the northern NCC during the Late Triassic implies that the lithospheric mantle beneath the northern NCC was previously metasomatized by melts/fluids. Bailinchuan Late Triassic syenites were formed in a post-collisional extensional setting, which provides time constraints on the major geodynamic setting at the northern NCC.
\end{abstract}

Keywords: LA-ICP-MS zircon U-Pb dating; alkaline rocks; petrogenesis; tectonic implications; Liaodong Peninsula

\section{Introduction}

Syenites are commonly intimately associated with alkaline mafic rocks, especially alkali to transitional basalts [1,2]. Numerous petrological, geochemical and experimental studies have suggested that the lithosphere mantle $[3,4]$ and crust $[5,6]$ are possible sources for syenites. The North China Craton (NCC) is known for its many alkaline intrusions, forming an east-west trending alkaline magmatic belt in northern NCC [7-9]. Liaodong Peninsula, located in the northeastern part of the NCC, is one of the important areas where the Mesozoic granitoid rocks are widely distributed. Bailinchuan alkaline syenite (BAS) is a representative pluton and located in the easternmost part of the belt [10-12]. This alkaline rock, contains valuable information concerning geodynamic processes. Previous studies have focused on the mineralogy, geochemistry, and geochronology $[9,12]$. Detailed petrogenesis, and tectonic environments of BAS still remain a controversial issue, three models for tectonic setting 
have been proposed, i.e., post-orogenic, rift or intraplate settings. This is an important scientific question in the study of Late Triassic tectonic evolution of the Liaodong peninsula. Besides, there are two prevailing views regarding the age of BAS: (1) The Rb-Sr isochron age of BAS was $218 \pm 39 \mathrm{Ma}$, while the errors were large and difficult to constrain the exact age [11]; (2) the age of this alkaline rock, was dated by zircon LA-ICP-MS U-Pb age at $217 \mathrm{Ma}$ [13]. However, they did not give specific petrology data and discuss tectonic events. Moreover, Jing et al. suggested that lithosphere mantle were possible sources for BAS [14]; Tan et al. proposed that BAS magma would be formed from the mixing of magma produced from the metasomatized upper mantle with the magma produced from the metasomatized crust in different ratios [15]; Yan et al. believed that the development of the BAS alkaline magmatism on the northern margin of the NCC is related to the extensional tectonic regime [11]. The abovementioned disagreement and deficiency call for further systematic research. Herein, we focus on the BAS, report the zircon $\mathrm{U}-\mathrm{Pb}$ age, petrological, geochemical data as well as $\mathrm{Lu}-\mathrm{Hf}$ isotopic results, aiming to provide constraints on (1) condition of zircon formation; (2) formation age of BAS; (3) the petrogenesis and mantle source characteristics; (4) tectonic setting of the BAS.

\section{Regional Geology and Petrography}

\subsection{Regional Geology}

The NCC is bound between the Paleo-Asian Orogenic Belt in the north and Qinling-Dabie-Sulu ultrahigh-pressure metamorphic belt in the south [16]. The NCC is considered to have been amalgamated between 1.8 and $1.9 \mathrm{Ga}$ along the Middle Trans-North China Orogen between the Eastern and Western Blocks [17-19]. NCC has been reactivated since the Mesozoic, featured by voluminous magmatic activities. The northeast part of NCC was impacted by three major tectonic events since the Mesozoic, namely the Central Asian orogenic belt on its north margin, the Dabie-Sulu orogenic belt on its eastern segment and the subduction of Pacific plate on its eastern margin (Figure 1A). The geological and geochronological data show that during Permian to Middle Triassic time, the NCC collided with the terranes in the Paleo-Asian Ocean in the north, and with the Yangtze craton in the south (e.g., $[15,17])$. Decratonization of the NCC was induced by these major plate tectonic processes since Mesozoic time (e.g., [18-21]). The tectonic setting of Liaodong peninsula suggests that this region could be spatially under the influence of three major tectonic domains: (1) to the east is Circle Pacific domain, subjected to Palaeo-Pacific plate subduction; (2) to the south is Dabie-Sulu orogenic belt, associated with the subduction and subsequent collision between Yangtze Craton and North China Craton; (3) to the north is Central Asian orogenic belt, representing the closure of Palaeo-Asian Ocean and collision of an amalgamated North China-Mongolian plate with the Siberian plate. Temporally the subduction of Palaeo-Pacific plate took place in Early and Middle Jurassic. The final closure of the Palaeo-Asian Ocean and amalgamation between the Mongolian arc terranes and North China block is generally thought to take place at Late Permian to Early Triassic. In the Liaodong Peninsula, about $20,000 \mathrm{~km}^{2}$ of Late Mesozoic intrusive rocks have been identified (Figure 1B), along with minor volcanic rocks. These intrusions can be mainly divided into three groups: (1) Jurassic (180-153 Ma) tonalite, diorite and gneissic two-mica monzogranite that have experienced ductile deformation, and undeformed to slightly deformed, represented by the paleo-Pacific slab has subducted beneath Eurasian continent. (2) Early Cretaceous (131-120 Ma) diorite, granodiorite, monzogranite and syenogranite [22], represented by a lithospheric extensional setting. In addition, minor Triassic magmatism (230-210 Ma) has been identified in the southern Liaodong Peninsula, consisting of mafic dikes, syenites, diorites and monzogranites with mafic enclaves, and they represent a post-collisional extension in Late Triassic [22,23]. Many alkaline intrusions occur in the northern NCC, forming an east-west trending alkaline magmatic belt $[10,23]$. Age data indicate that the alkaline intrusions were emplaced in the Triassic $[8,10,24]$. Details of tectonic settings and geodynamic evolutions of the NCC that have been described $[10,16,24]$. Multi-stage rifting events occurred in the period from Late Paleoproterozoic to Neoproterozoic after the cratonization of the NCC $[25,26]$. 
Major orogenic events occurred from the Paleozoic to Mesozoic along the northern margin of the NCC. The final closure of oceans and large scale collision between the Yangtze and North China continents during the period of Triassic to Late Jurassic (240-140 Ma) occurred within transcontinental slab stacking, thickening and crustal shortening.

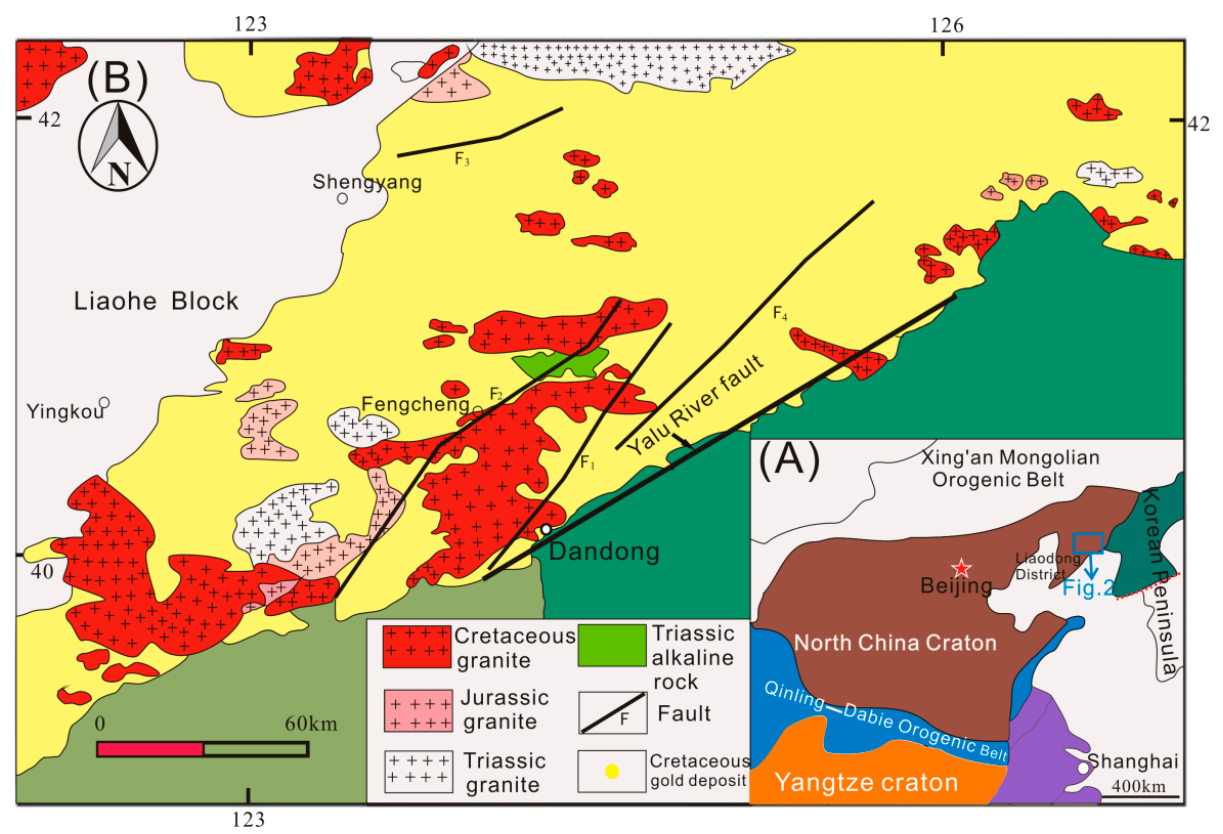

Figure 1. (A) Simplified geological map of eastern China, showing major tectonic units; rectangle outlines the study area; (B) geological map showing distribution of Mesozoic intrusions in the Liaodong Peninsula [12].

\subsection{Petrography}

BAS consists of alkaline volcanics and alkaline intrusive rocks (Figure 2). The volcanic rocks are located within the central part of the BAS. They are composed of trachytes and minor phonolites. Most of them are covered by Jurassic sedimentary rocks. The alkaline intrusive rocks are composed of grass-green aegirine-nephelinesyenites and alkaline syenites (Figure 3).

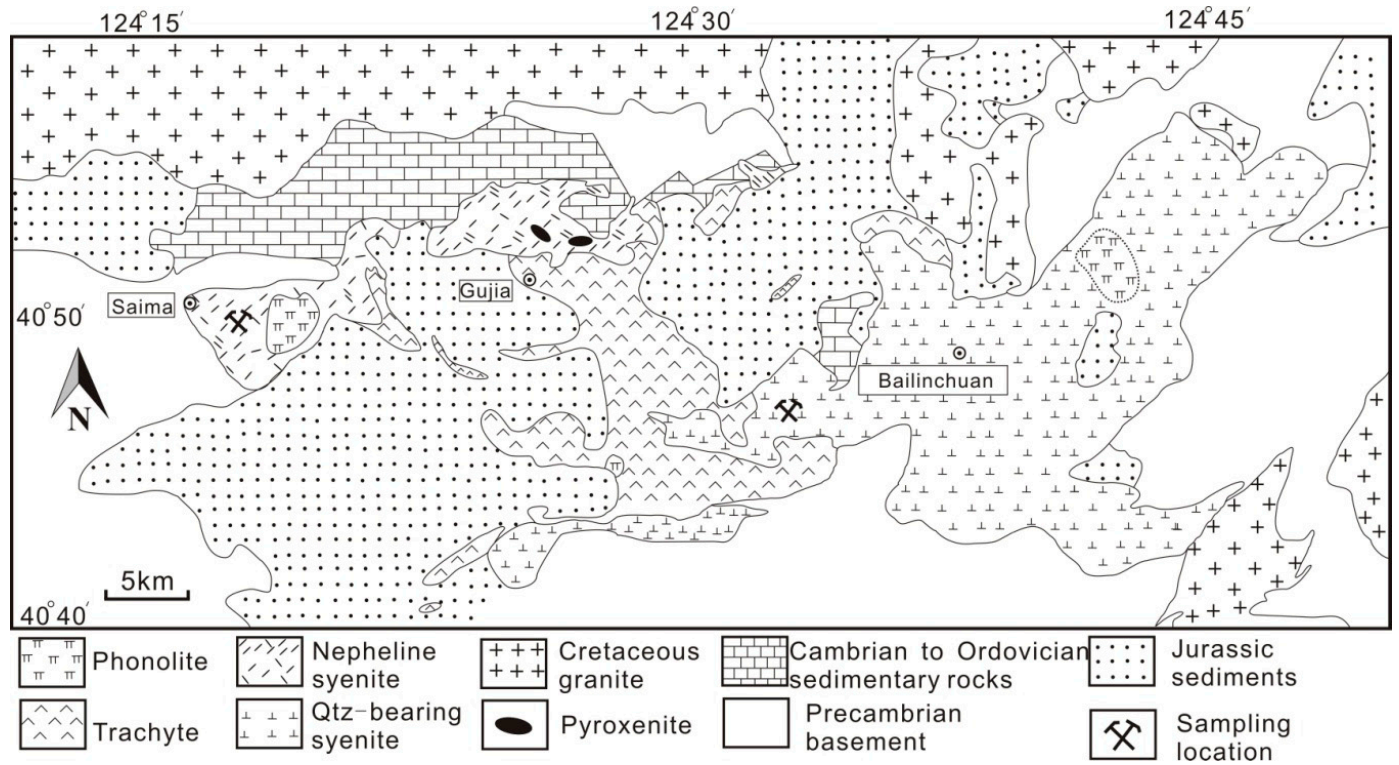

Figure 2. Geological map of the Bailinchuan alkaline complex (Modified after [9]). 

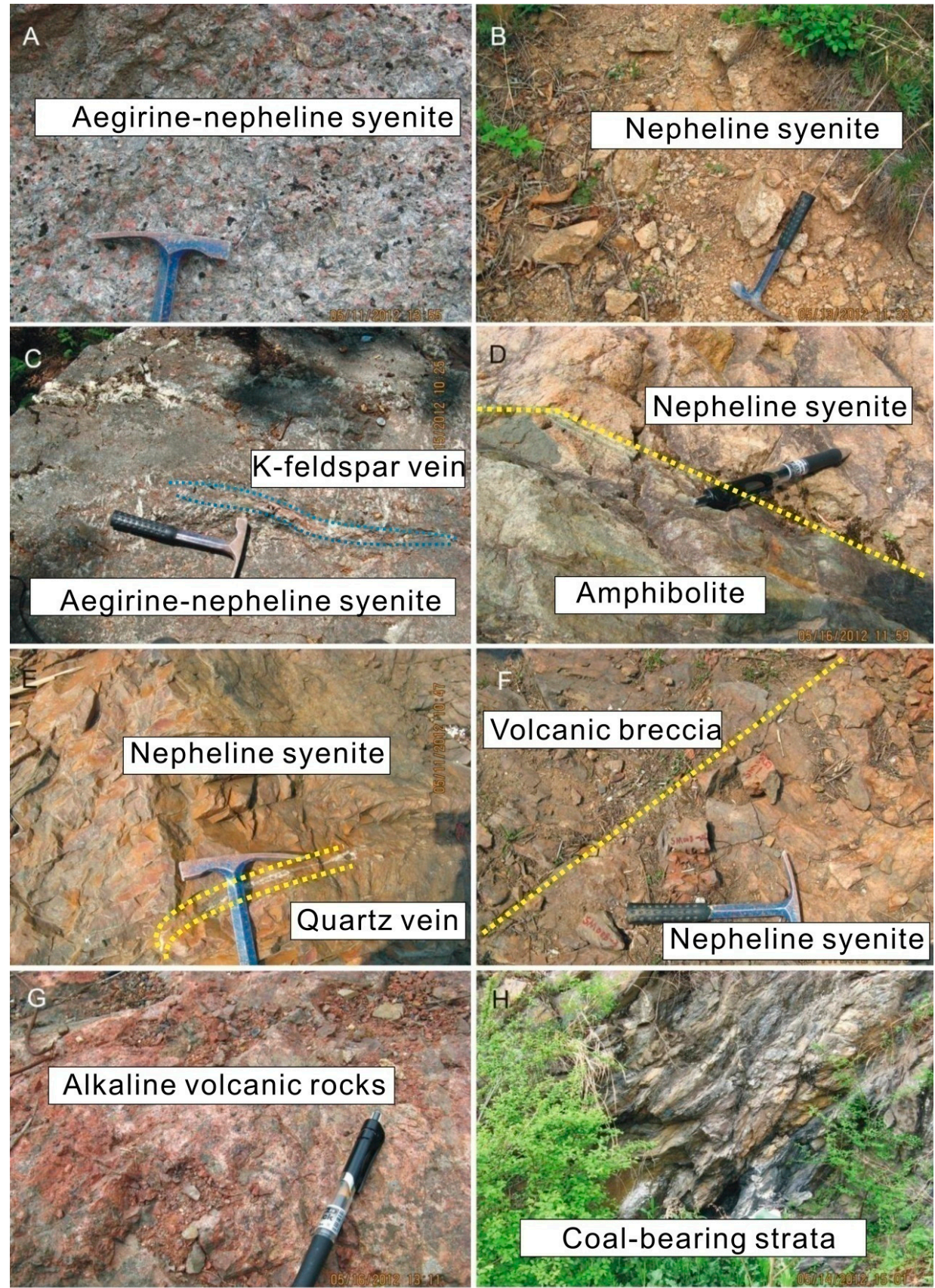

Figure 3. Field photographs of Bailinchuan alkaline complex. (A) Coarse grain aegirine-nepheline syenite; (B) An out crop of nepheline syenite; (C) K-feldspar vein occur in aegirine-nepheline syenite; (D) The contact zone between amphibolite and alkaline syenite; (E) Carbonate veins occur in nepheline syenite; (F) The contact zone between volcanic breccia and alkaline syenite; (G) Alkaline volcanic rock; (H) An out crop of coal bearing strata.

\subsubsection{Aegirine-Nephelinesyenite}

These kinds of samples are collected from Saima. Aegirine-nephelinesyenite mainly occurs in the western part of the area, near Saima. Aegirine-nephelinesyenites are grey to light pink, fine- to medium-grained, mainly composed of alkaline feldspar $(\sim 70-75 \%)$, nepheline $(\sim 20-25 \%)$, and aegirine $(\sim 8 \%)$, as well as minor accessory sphene and magnetite. The alkaline feldspar are subhedral to euhedral, medium- to coarse- grained (Figure 4). The aegirines are subhedral to euhedral, grass-green, and some of them have a radial shape. Euhedral nepheline usually occurs in the alkali feldspar, while the anhedral nepheline is large and exists within the interstitial spaces between 
feldspars, suggesting that they are late-stage phases. Anhedral alkali feldspar surrounds the aegirine and nepheline.

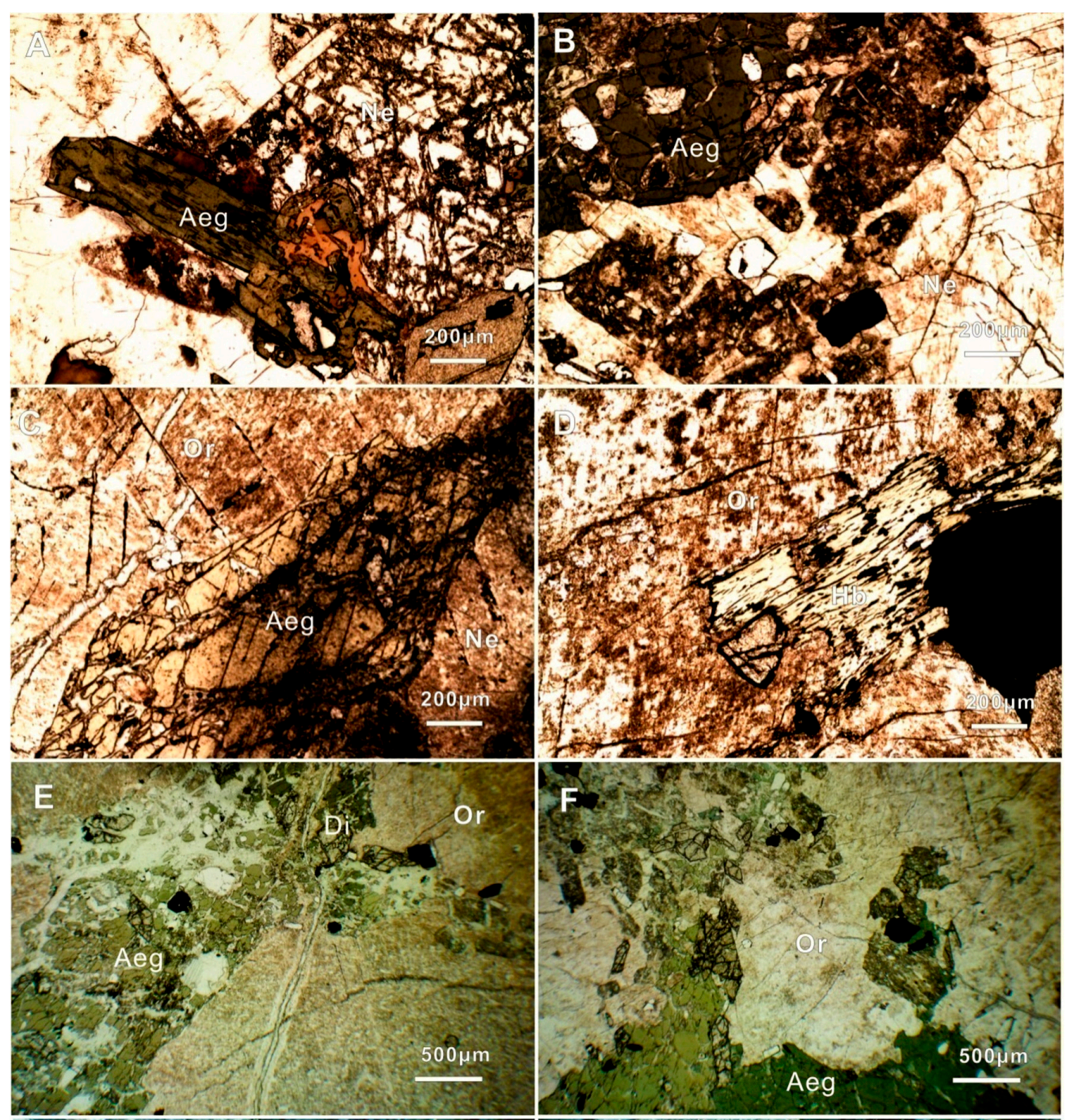

Figure 4. Thin section photomicrographs of $(\mathbf{A}, \mathbf{B})$ mosaic textures with the assemblage aegirine + nepheline + orthoclase of aegirine-nepheline syenite, nepheline was subjected to alteration. (C) Aegirine-nepheline syenite with porphyric texture, consisting of aegirite, nepheline, orthoclase. (D) Mosaic texture with the orthoclase + hornblende of alkaline syenite. Abbreviations are Aeg: aegirine; Di: diopside; $\mathrm{Hb}$ : hornblende; Ne: nepheline; Or: orthoclase. (E) Aegirite bonded with diopside in the aegirine-nepheline syenite, consisting of aegirite, nepheline, orthoclase. (F) alkaline syenite, consisting of orthoclase, oligoclase was subjected to low sericitization alterted.

\subsubsection{Alkaline Syenite}

These kinds of samples are collected from Bailinchuan. Alkaline syenite mainly occurs in the eastern part of the area. In addition, minor aplite is sparsely distributed. Based on petrographic observations, the mineral assemblage mainly consists of alkaline feldspar ( 80\%) and plagioclase $(\sim 10 \%)$ (Figure 4$)$. The grain sizes of feldspar vary from 0.05 to $0.5 \mathrm{~mm}$. The melanocratic mineral mainly consists of alkaline pyroxene $(\sim 3 \%)$ and biotite $(\sim 7 \%)$. The rare euhedral-subhedral crystals are compositionally sphene, zircon and magnetite. The alkaline feldspar are subhedral to euhedral, medium- to coarse-grained. The plagioclase displays sericitization and forms euhedral grains better than alkali feldspar. The plagioclase also shows polysynthetic twinning and zonal structures. The alkali 
feldspar is fresh and is mainly composed of orthoclase and microcline. The crystals are tabular and hypidiomorphic-euhedral, with Carlsbad twins and gridiron twins under crossed polarized light. Biotite occurs in minor amount $(\sim 7 \%)$ and is small in size $(<0.3 \mathrm{~mm})$, varying from yellowish-brown to dark-brown. The alkaline pyroxenes are subhedral to euhedral, pale-green, and some have darker green margins.

\section{Samples and Analytical Methods}

\subsection{U-Pb Dating and In Situ Trace Element Analysis of Zircon}

Rock samples selected for this study include two alkaline syenites (BLC-1, BLC-2), as shown by their field photographs and microstructures as above, and in specific sampling localities are shown in Figure 2. The zircon grains for $\mathrm{U}-\mathrm{Pb}$ analysis were mounted in epoxy mounts that were then polished to section the crystals in half for analysis. All zircons were documented using transmitted and reflected light photomicrographs and cathodoluminescence (CL) images to reveal their internal structures. Uranium- $\mathrm{Th}-\mathrm{Pb}$ ratios analyses in this study were performed using laser ablation-inductively coupled plasma-mass spectrometry LA-ICP-MS at the State Key Laboratory of Continental Dynamics, Northwest University, China. The detailed analytical procedures of the LA-ICP-MS zircon U-Pb dating are described by [27]. This instrument set-up includes an ArFexcimer laser ablation system (Geolas CQ) attached to an Agilent 7500a ICP-MS instrument. The analyses were carried out with a beam diameter of $60 \mu \mathrm{m}$, a repetition rate of 3-10 Hz, and an energy of $\sim 10 \mathrm{~J} / \mathrm{cm}^{2}$. During laser ablation, helium gas was flushed into the sample cell to minimize aerosol deposition around the ablation pit, and argon gas was then used to improve transport efficiency. The background of ${ }^{204} \mathrm{~Pb}$ and ${ }^{202} \mathrm{Hg}$ was less than $100 \mathrm{cps}$ because of the use of high-purity liquid argon and helium gas, compared to the range of 10 to $500 \mathrm{cps}$ for zircon depending on its common Pb contents. The ICP-MS measurements were carried out using time-resolved analysis and peak hopping at one point per mass. Every eight samples analyzed were followed by one standard zircon 91500 and one NIST SRM 610 measurement. The ${ }^{207} \mathrm{~Pb} /{ }^{206} \mathrm{~Pb},{ }^{206} \mathrm{~Pb} /{ }^{238} \mathrm{U},{ }^{207} \mathrm{U} /{ }^{235} \mathrm{U}$ and ${ }^{208} \mathrm{~Pb} /{ }^{232} \mathrm{Thratios}$ were corrected by using zircon 91500 as an external standard. The raw ICP-MS data were exported in ASCII format and processed using GLITTER 4.0. The relative standard deviations of the reference values for zircon 91500 were set at $2 \%$. After obtaining the Pb isotopic ratios. Subsequently, ${ }^{206} \mathrm{~Pb} /{ }^{238} \mathrm{U}$ weighted ages were calculated using ISOPLOT 3.0 [27].

\subsection{Zircon Hf Isotope Analyses}

The Geolas 2005 excimer ArF laser-ablation system (MicroLas ${ }^{\mathrm{TM}}$ Beam Delivery Systems, Lambda Physik AG, Gttingen, Germany) coupled with a Nu Plasma HRMC-ICP-MS (Nu Instruments Ltd., Wrexham, UK) were used to measure the Hf isotopes at the State Key Laboratory of Continental Dynamics in Northwest University, Xi'an with a spot size of $44 \mu \mathrm{m}$. Helium was applied as carrier gas to provide efficient aerosol transport to the ICP and minimize aerosol deposition. The detailed analytical methods were the same as those described by [27]. Data are processed using a Hf calculate program-8.6 provided by the laboratory. Standard zircons MON-1, GJ-1 and 91500 were analyzed every $10-15$ zircon grains, yielded mean ${ }^{176} \mathrm{Hf} /{ }^{177} \mathrm{Hf}$ isotopic ratios of $0.282731 \pm 16(2 \sigma)$ for MON-1, $0.282013 \pm 18(2 \sigma)$ for GJ-1, $0.282295 \pm 18(2 \sigma)$ for 91,500, which are in good agreement with the recommended values of $0.282738 \pm 8(2 \sigma)(\mathrm{MON}-1), 0.282015 \pm 19(2 \sigma)(\mathrm{GJ}-1), 0.282307 \pm 58(2 \sigma)$ (91500).

\subsection{Major and Trace Element Analyses}

We selected alkaline syenite samples to carry out major and trace element analysis. These samples were the same as those used for $\mathrm{U} / \mathrm{Pb}$ dating. Whole-rock samples were trimmed to remove altered surfaces, cleaned with de-ionized water, and then crushed and powdered using an agate mill. The major element compositions were analyzed using a Philips (Philips PW2404, Amsterdam, The Netherlands) 
X-ray fluorescence spectrometer (XRF) at the Beijing Research Institute of Uranium Geology, China National Nuclear Corporation. The test methods were followed by GB/T 14506-2010 and had a precision that was better than 3\%. Trace element concentrations were determined by inductively coupled plasma mass spectrometry (ICP-MS) using an Agilent 7500a system. About $50 \mathrm{mg}$ of crushed whole-rock powders was dissolved using $0.01 \mathrm{~mL} \mathrm{HClO}_{4}, 1.5 \mathrm{~mL} \mathrm{HF}$ and $1.5 \mathrm{~mL} \mathrm{HNO}_{3}$ mixtures in a Teflon bomb which was placed on the hot plate at $140{ }^{\circ} \mathrm{C}$. This was followed by evaporation to dryness, refluxing with $1.5 \mathrm{~mL} \mathrm{HF}$ and $1.5 \mathrm{~mL} \mathrm{HNO}_{3}$ before being sealed with a cap and placed in a steel jacket, and then placed in an oven at $195{ }^{\circ} \mathrm{C}$ for $48 \mathrm{~h}$. The next step was evaporating the solution to dryness, dissolving it with $3 \mathrm{~mL} \mathrm{HNO}_{3}$, and drying it again. Then, $3 \mathrm{~mL} \mathrm{HNO}_{3}$ was added before it was again sealed with a cap and placed in a steel jacket, and replaced in the oven at $140{ }^{\circ} \mathrm{C}$ for $12 \mathrm{~h}$. Finally, the solution was extracted after cooling, and then diluted to $100 \mathrm{~g}$. $1 \mathrm{~g}$ rhodium was added into the diluted solution as an internal standard to monitor drift in mass response during mass spectrometric measurements. The analytical uncertainties are about $2-5 \%$ for the trace elements. Trace elements in zircon were analyzed by LA-ICP-MS. Analytical uncertainties for LA-ICP-MS trace element analyses are $\pm 10 \%$ and ca. $\pm 5 \%$ for elements with abundances lower and higher than 10 ppm, respectively [28].

\section{Results}

\subsection{Zircon Morphology, U-Pb Dating}

To date the emplacement ages of these alkaline rocks, two alkaline syenites samples were chosen for zircon $\mathrm{U}-\mathrm{Pb}$ dating by LA-ICP-MS. The $\mathrm{U}-\mathrm{Pb}$ results are listed in Table 1 and the CL images are shown in Figure 5. Most of the zircons from the syenites have a size of 50-160 $\mu \mathrm{m}$ with a length/width ratio of 1:1 to 3:1 and show oscillatory growth zoning in CL images with more homogeneous patches (Figure 5), suggesting a magmatic origin. Fifteen spots were analyzed from sample BLC-1. They have indistinguishable ${ }^{206} \mathrm{~Pb} /{ }^{238} \mathrm{U}$ ratios within analytical uncertainty, corresponding to a single age population with a weighted mean ${ }^{206} \mathrm{~Pb} /{ }^{238} \mathrm{U}$ age of $229 \pm 3 \mathrm{Ma}(\mathrm{MSWD}=0.75)$, which is interpreted to be the crystallization age (Figure 6A). Fourteen spots were analyzed from sample BLC-2. All the analyses are concordant in terms of ${ }^{206} \mathrm{~Pb} /{ }^{238} \mathrm{U}$ and ${ }^{207} \mathrm{~Pb} /{ }^{235} \mathrm{~Pb}$ within analytical errors (Figure $6 \mathrm{~B}$ ). The results yield a weighted mean ${ }^{206} \mathrm{~Pb} /{ }^{238} \mathrm{U}$ age of $226 \pm 2 \mathrm{Ma}(\mathrm{MSWD}=1.40)$, which is interpreted to be the crystallization age.
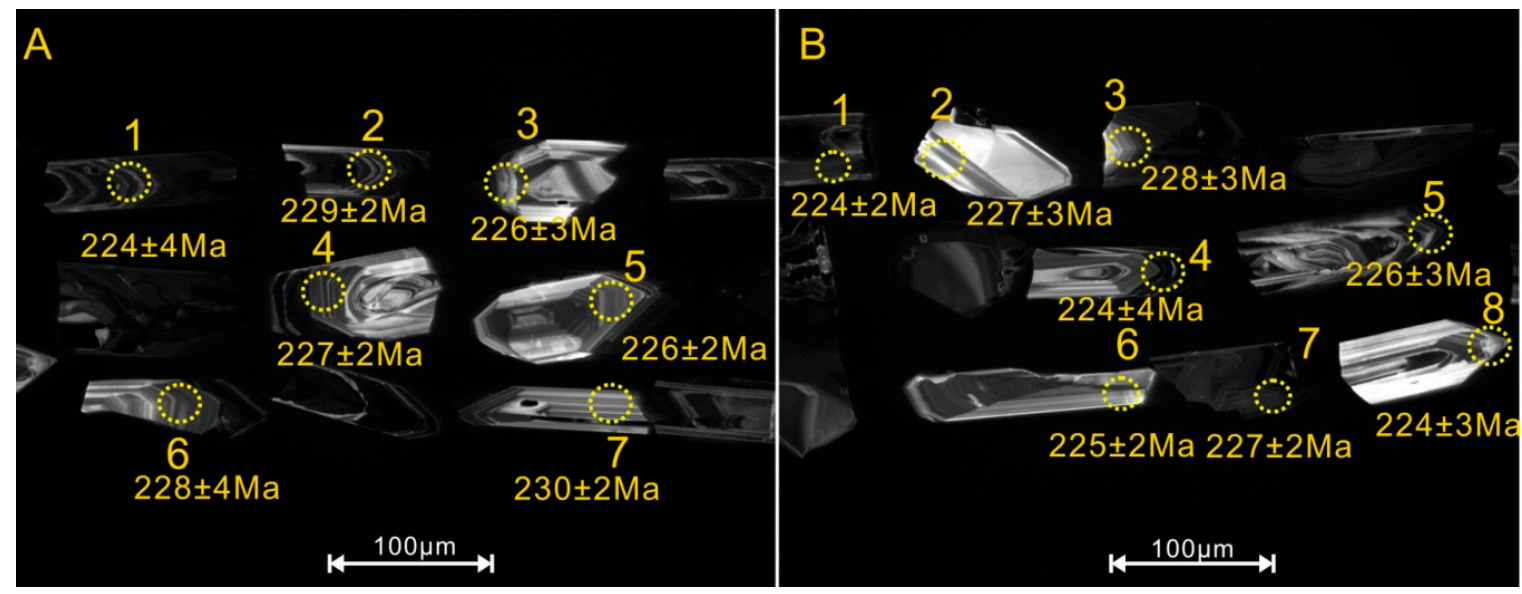

Figure 5. Cathodoluminescence images of zircons from the BAS. (A) BLC-1and (B) BLC-2. 

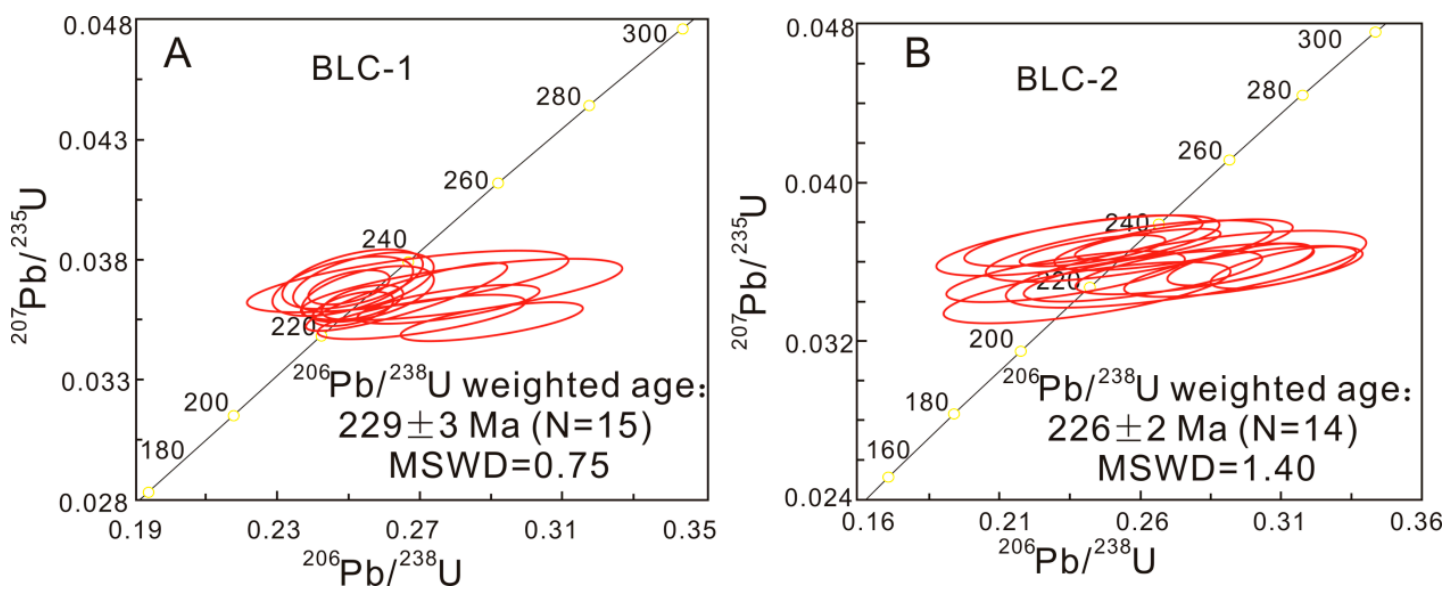

Figure 6. Zircon LA-ICP-MS U-Pb concordia diagrams for (A) BLC-1 and (B) BLC-2. The weighted mean age and MSWD are shown in each figure.

\subsection{Zircon Lu-Hf Isotopes and Geochemistry}

\subsubsection{Zircon Lu-Hf Isotopes}

Zircon Lu-Hf isotope concentrations were determined for 15 points from sample BLC-1 on the same or similar domains of the zircon grains dated for this study. Calculation of $\varepsilon \mathrm{Hf}(\mathrm{t})$ values was based on the present chondritic uniform reservoir (CHUR) values with ${ }^{176} \mathrm{Hf} /{ }^{177} \mathrm{Hf}=0.282785$ and ${ }^{176} \mathrm{Lu} /{ }^{177} \mathrm{Hf}=0.0336$, using the decay constant of ${ }^{176} \mathrm{Lu}$ of $1.867 \times 10^{-11}$ year $^{-1}$ [29]. Results of the $\mathrm{Lu}-\mathrm{Hf}$ isotope measurements are given in Table 1 . Initial ${ }^{176} \mathrm{Hf} /{ }^{177} \mathrm{Hf}$ ratios range from 0.281926 to 0.282051 , with $\varepsilon \mathrm{Hf}(\mathrm{t})$ values of -20.8 to -16.0 , indicating formation from a slightly depleted mantle source. The ${ }^{176} \mathrm{Lu} /{ }^{177} \mathrm{Hf}$ ratios scatter moderately from 0.000435 to 0.000924 , which yielded ananomalously high ${ }^{176} \mathrm{Lu} /{ }^{177} \mathrm{Hf}$ ratio of 0.000651 . The $\mathrm{Lu}-\mathrm{Hf}$ isotope data do not for many distinct clusters and show no correlation with the analyzed zircon domain. There is no obvious correlation between the Lu-Hf data and their associated ${ }^{206} \mathrm{~Pb} /{ }^{238} \mathrm{U}$ ages. This might be due to the fact that the laserspots for $\mathrm{U}-\mathrm{Pb}$ and $\mathrm{Lu}-\mathrm{Hf}$ analyses did not overlap. Hf model ages have a narrow range from $\mathrm{T}_{\mathrm{DM}}=2.4$ to $\mathrm{T}_{\mathrm{DM}}=2.6 \mathrm{Ga}$. Model ages are older than the associated ${ }^{206} \mathrm{~Pb} /{ }^{238} \mathrm{U}$ ages.

The analytical results are listed in Table 2. They show relatively homogeneous Hf isotopic compositions, with initial ${ }^{176} \mathrm{Hf} /{ }^{177} \mathrm{Hf}$ ratios of $0.281926-0.282051, \varepsilon \mathrm{Hf}(\mathrm{t})$ values of -20.8 to -16.3 . All analyzed spots plot between the lower crust and the average crust lines on the $\varepsilon H f(t) v s . t$ diagram (Figure 7).

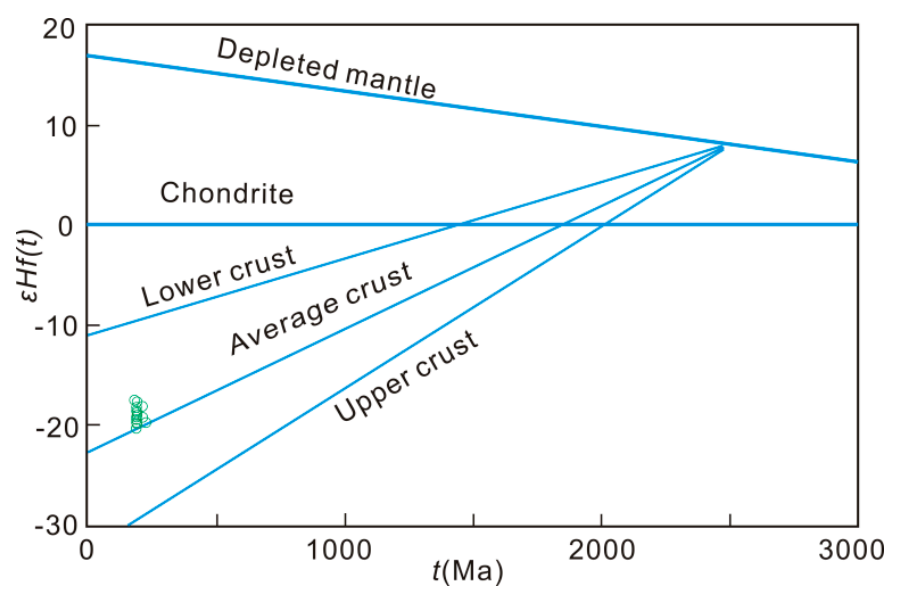

Figure 7. Plots of $\varepsilon \mathrm{Hf}(\mathrm{t})$ vs. age of zircons. The UCC (upper continental crust) and LCC (lower continental crust) are from Jahn et al. [29]. 
Table 1. Laser ablation inductively coupled plasma mass spectrometry (LA-ICP-MS) zircon U-Pb data for samplesBLC-1 and BLC-2, respectively.

\begin{tabular}{|c|c|c|c|c|c|c|c|c|c|c|}
\hline \multirow{2}{*}{$\begin{array}{c}\text { BLC-1 } \\
\text { Plot }\end{array}$} & \multicolumn{6}{|c|}{ U-Pb Isotope Ratios } & \multicolumn{4}{|c|}{ Isotopic Ages (Ma) } \\
\hline & ${ }^{207} \mathrm{~Pb} /{ }^{206} \mathrm{~Pb}$ & $1 \sigma$ & ${ }^{207} \mathrm{~Pb} /{ }^{235} \mathrm{U}$ & $1 \sigma$ & ${ }^{206} \mathrm{~Pb} /{ }^{238} \mathrm{U}$ & $1 \sigma$ & ${ }^{207} \mathrm{~Pb}^{/ 235} \mathrm{U}$ & $1 \sigma$ & ${ }^{206} \mathrm{~Pb} /{ }^{238} \mathrm{U}$ & $1 \sigma$ \\
\hline 1 & 0.05339 & 0.00405 & 0.25472 & 0.01783 & 0.03460 & 0.00077 & 230 & 14 & 219 & 5 \\
\hline 2 & 0.04997 & 0.00207 & 0.26208 & 0.00842 & 0.03803 & 0.00063 & 236 & 15 & 241 & 4 \\
\hline 3 & 0.07156 & 0.00267 & 0.39660 & 0.01059 & 0.04019 & 0.00066 & 224 & 5 & 226 & 4 \\
\hline 4 & 0.05171 & 0.00277 & 0.26170 & 0.01211 & 0.03670 & 0.00066 & 236 & 10 & 232 & 4 \\
\hline 5 & 0.05121 & 0.00184 & 0.25579 & 0.00633 & 0.03622 & 0.00058 & 231 & 5 & 229 & 4 \\
\hline 6 & 0.04964 & 0.00247 & 0.25864 & 0.01083 & 0.03778 & 0.00066 & 234 & 9 & 239 & 4 \\
\hline 7 & 0.05029 & 0.00190 & 0.25350 & 0.00690 & 0.03655 & 0.00059 & 229 & 6 & 231 & 4 \\
\hline 8 & 0.05361 & 0.00296 & 0.27736 & 0.01332 & 0.03752 & 0.00070 & 224 & 11 & 217 & 4 \\
\hline 9 & 0.12023 & 0.00588 & 0.62816 & 0.02507 & 0.03789 & 0.00077 & 230 & 7 & 228 & 5 \\
\hline 10 & 0.05184 & 0.00276 & 0.25305 & 0.01161 & 0.03540 & 0.00064 & 229 & 9 & 224 & 4 \\
\hline 11 & 0.05515 & 0.00212 & 0.28048 & 0.00784 & 0.03688 & 0.00060 & 231 & 6 & 233 & 4 \\
\hline 12 & 0.05024 & 0.00258 & 0.24359 & 0.01061 & 0.03516 & 0.00063 & 221 & 9 & 223 & 4 \\
\hline 13 & 0.05912 & 0.00268 & 0.28561 & 0.01041 & 0.03503 & 0.00061 & 225 & 8 & 222 & 4 \\
\hline 14 & 0.05162 & 0.00232 & 0.25445 & 0.00917 & 0.03574 & 0.00061 & 230 & 7 & 226 & 4 \\
\hline 15 & 0.06982 & 0.00595 & 0.33529 & 0.02654 & 0.03482 & 0.00089 & 222 & 6 & 216 & 6 \\
\hline 16 & 0.04788 & 0.00135 & 0.24964 & 0.00665 & 0.03776 & 0.00031 & 226 & 5 & 239 & 2 \\
\hline 17 & 0.06324 & 0.00254 & 0.34950 & 0.00440 & 0.03646 & 0.00019 & 234 & 3 & 221 & 1 \\
\hline 18 & 0.05385 & 0.00129 & 0.26548 & 0.00589 & 0.03572 & 0.00027 & 239 & 5 & 226 & 2 \\
\hline 19 & 0.05450 & 0.00182 & 0.26523 & 0.00843 & 0.03527 & 0.00034 & 239 & 7 & 223 & 2 \\
\hline 20 & 0.04487 & 0.00163 & 0.22340 & 0.00776 & 0.03610 & 0.00034 & 205 & 6 & 229 & 2 \\
\hline 21 & 0.04881 & 0.00107 & 0.23988 & 0.00483 & 0.03564 & 0.00025 & 218 & 4 & 226 & 2 \\
\hline 22 & 0.04498 & 0.00219 & 0.23436 & 0.01107 & 0.03779 & 0.00045 & 214 & 9 & 239 & 3 \\
\hline 23 & 0.05358 & 0.00224 & 0.26614 & 0.01068 & 0.03604 & 0.00041 & 240 & 9 & 228 & 3 \\
\hline 24 & 0.05040 & 0.00179 & 0.24667 & 0.00839 & 0.03551 & 0.00034 & 224 & 7 & 225 & 2 \\
\hline 25 & 0.04912 & 0.00192 & 0.23941 & 0.00899 & 0.03537 & 0.00036 & 218 & 7 & 224 & 2 \\
\hline 26 & 0.04935 & 0.00188 & 0.24097 & 0.00882 & 0.03544 & 0.00036 & 219 & 7 & 225 & 2 \\
\hline 27 & 0.05131 & 0.00207 & 0.25455 & 0.00986 & 0.03601 & 0.00038 & 230 & 8 & 228 & 2 \\
\hline 28 & 0.04943 & 0.00179 & 0.25313 & 0.00877 & 0.03717 & 0.00036 & 229 & 7 & 235 & 2 \\
\hline 29 & 0.04903 & 0.00166 & 0.24386 & 0.00790 & 0.03610 & 0.00033 & 222 & 6 & 229 & 2 \\
\hline
\end{tabular}


Table 2. Zircon Lu-Hf isotopic compositions of the Bailinchuansyenite.

\begin{tabular}{|c|c|c|c|c|c|c|c|c|}
\hline Spot & Age (Ma) & ${ }^{176} \mathrm{Hf} /{ }^{177} \mathrm{Hf}$ & ${ }^{176} \mathrm{Lu} /{ }^{177} \mathrm{Hf}$ & ${ }^{176} \mathrm{Yb} /{ }^{177} \mathrm{Hf}$ & $\left({ }^{176} \mathrm{Hf} /{ }^{177} \mathrm{Hf}\right)_{t}$ & $\varepsilon \mathbf{H f}(\mathbf{0})$ & $\varepsilon H f(t)$ & $\begin{array}{c}\mathrm{T}^{\mathrm{C}} \\
\mathrm{DM}(\mathrm{Ma})\end{array}$ \\
\hline BLC-1-1 & 229 & 0.282051 & 0.000623 & 0.025899 & 0.282025 & -22.6 & -16.3 & 2418 \\
\hline BLC-1-2 & 229 & 0.281956 & 0.000435 & 0.019210 & 0.282014 & -26.8 & -18.5 & 2606 \\
\hline BLC-1-3 & 229 & 0.281976 & 0.000821 & 0.034580 & 0.281894 & -25.9 & -18.1 & 2596 \\
\hline BLC-1-4 & 229 & 0.281937 & 0.000924 & 0.028759 & 0.281998 & -28.3 & -19.4 & 2625 \\
\hline BLC-1-5 & 229 & 0.281946 & 0.000726 & 0.026356 & 0.281959 & -29.6 & -20.2 & 2625 \\
\hline BLC-1-6 & 229 & 0.281998 & 0.000505 & 0.022734 & 0.281987 & -26.8 & -19.9 & 2621 \\
\hline BLC-1-7 & 229 & 0.281941 & 0.000614 & 0.026601 & 0.281939 & -28.8 & -20.5 & 2657 \\
\hline BLC-1-8 & 229 & 0.281972 & 0.000556 & 0.023946 & 0.281990 & -28.1 & -20.4 & 2649 \\
\hline BLC-1-9 & 229 & 0.282019 & 0.000659 & 0.025776 & 0.282134 & -27.7 & -18.9 & 2618 \\
\hline BLC-1-10 & 229 & 0.281971 & 0.000543 & 0.037629 & 0.281893 & -29.2 & -18.9 & 2618 \\
\hline BLC-1-11 & 229 & 0.281928 & 0.000648 & 0.026420 & 0.281899 & -28.3 & -19.8 & 2556 \\
\hline BLC-1-12 & 229 & 0.281972 & 0.000619 & 0.047589 & 0.281990 & -28.9 & -20.1 & 2618 \\
\hline BLC-1-13 & 229 & 0.281936 & 0.000682 & 0.019943 & 0.281965 & -29.1 & -20.8 & 2667 \\
\hline BLC-1-14 & 229 & 0.281939 & 0.000681 & 0.035783 & 0.281976 & -29.2 & -20.4 & 2631 \\
\hline BLC-1-15 & 229 & 0.281926 & 0.000732 & 0.031056 & 0.281941 & -29.7 & -20.7 & 2662 \\
\hline
\end{tabular}

Note: $\mathrm{Hf}$ mass bias correction to ${ }^{179} \mathrm{Hf} /{ }^{177} \mathrm{Hf}=0.7325 .{ }^{176} \mathrm{Lu} /{ }^{177} \mathrm{Hf}$ errors are $0.5 \%$. Reproducibility of JMC475 $\mathrm{Hf}$ standard over the period of analyses ${ }^{176} \mathrm{Hf} /{ }^{177} \mathrm{Hf}=0.282158 \pm 13(\mathrm{n}=6$ ) Decay constant $\lambda_{176 \mathrm{Lu}}=1.865 \times 10^{-11} \mathrm{yr}^{-1}$ adopted from [30]. Values used for $\varepsilon$ Hf calculations: ${ }^{176} \mathrm{Hf} /{ }^{177} \mathrm{Hf} \mathrm{CHUR}_{(0)}=0.282785$ and ${ }^{176} \mathrm{Lu} /{ }^{177} \mathrm{Hf} \mathrm{CHUR}(0)=0.0336[28]$. Depleted mantle model age calculations after [31] 


\subsubsection{Zircon Geochemistry}

Zircon LA-ICP-MS U-Pb ages and trace element data for BAS, all have similar age peaks and trace element compositions (Figure 8; Table 3). As such, all zircons yield moderate $U$ and Th contents with Th-U ratios of 0.49-3.84. In this case, the Th-U ratio is not correlated with age (Figure 8A) or texture of the analyzed zircon or zircon domain, respectively. Similarly, no clear correlation is found between the age and the concentrations of $\mathrm{U}$, Th or other trace elements (e.g., Y, Yb, Hf). Meanwhile, all zircons have broad concentrations of $\mathrm{P}$ (20-565.7 ppm), Y (53.3-3065.5 ppm) and $\mathrm{Nb}(0.1-9.3 \mathrm{ppm})$. Geochemical characteristics show that zircon has LREE/HREE ratio of (0.12-1.05). Provenance based on zircon geochemistry shows that zircon grains were derived from continental crust (Figure 8B). Chondrite normalized rare earth element (REE) patterns of zircons generally have enriched Ce values relative to $\mathrm{La}$ and $\mathrm{Pr}$, and depleted Eu values relative to Sm and Gd. Therefore, high Ce contents in zircon may imply oxidizing conditions $\left(\mathrm{Ce}^{4+}\right.$ is more compatible than $\left.\mathrm{Ce}^{3+}\right)$. The chondrite normalized REE patterns (Figure 9) indicate a similar chemical behavior of all zircons with a strong enrichment in HREE and a pronounced positive Ce anomaly. However, as for the Th-U ratio, there is no systematic change in the $\mathrm{Y}$ or REE content with age (Figure 8C). Interestingly, those analyses that yield a positive Eu anomaly exhibit a much lower positive Ce anomaly than analyses with no or a weak negative Eu anomaly (Figure 8D).
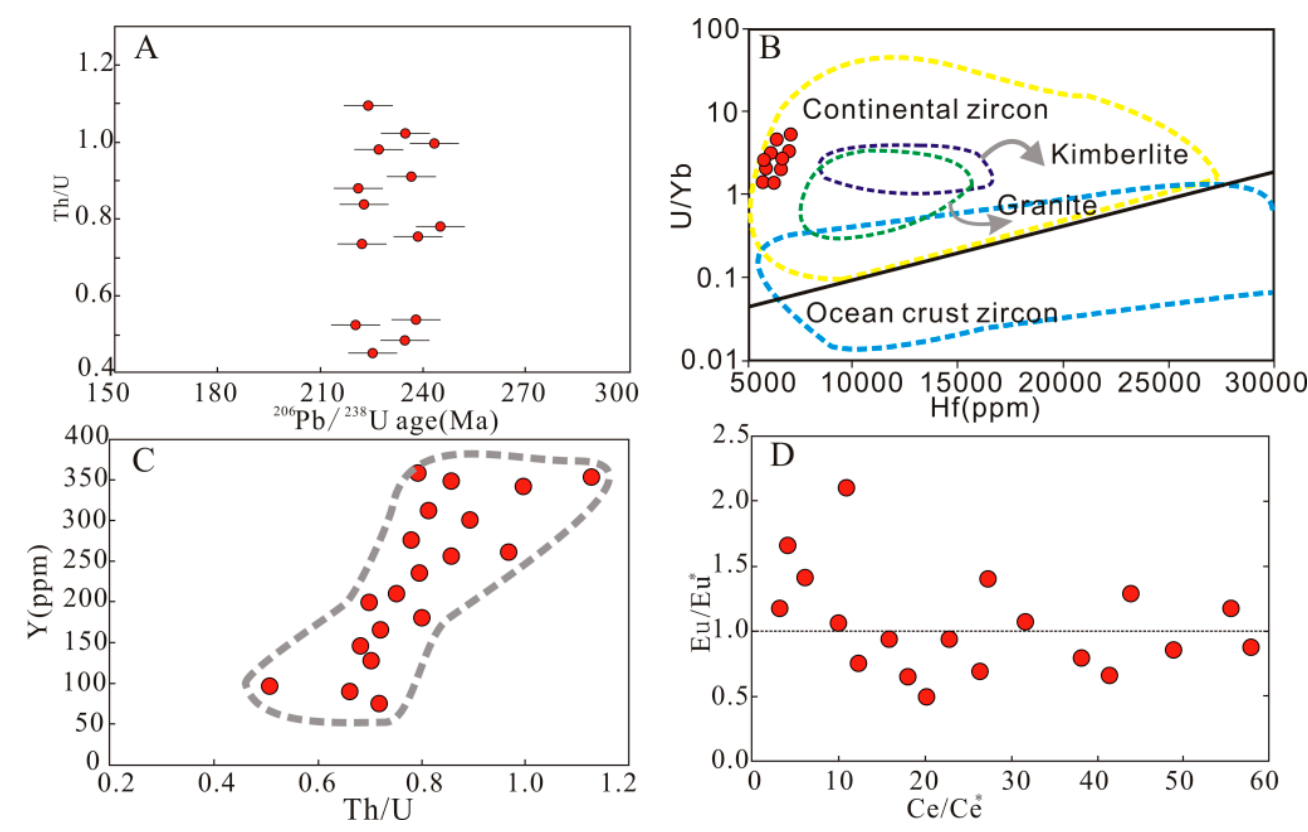

Figure 8. Plots of Th/U vs. U-Pb age (A), Hf concentration (in ppm) vs. U/Yb (B), Y concentration (in ppm) vs. Th/U (The initial data source: [32]) (C) and the relation between the measured Eu and Ce anomalies (D). 

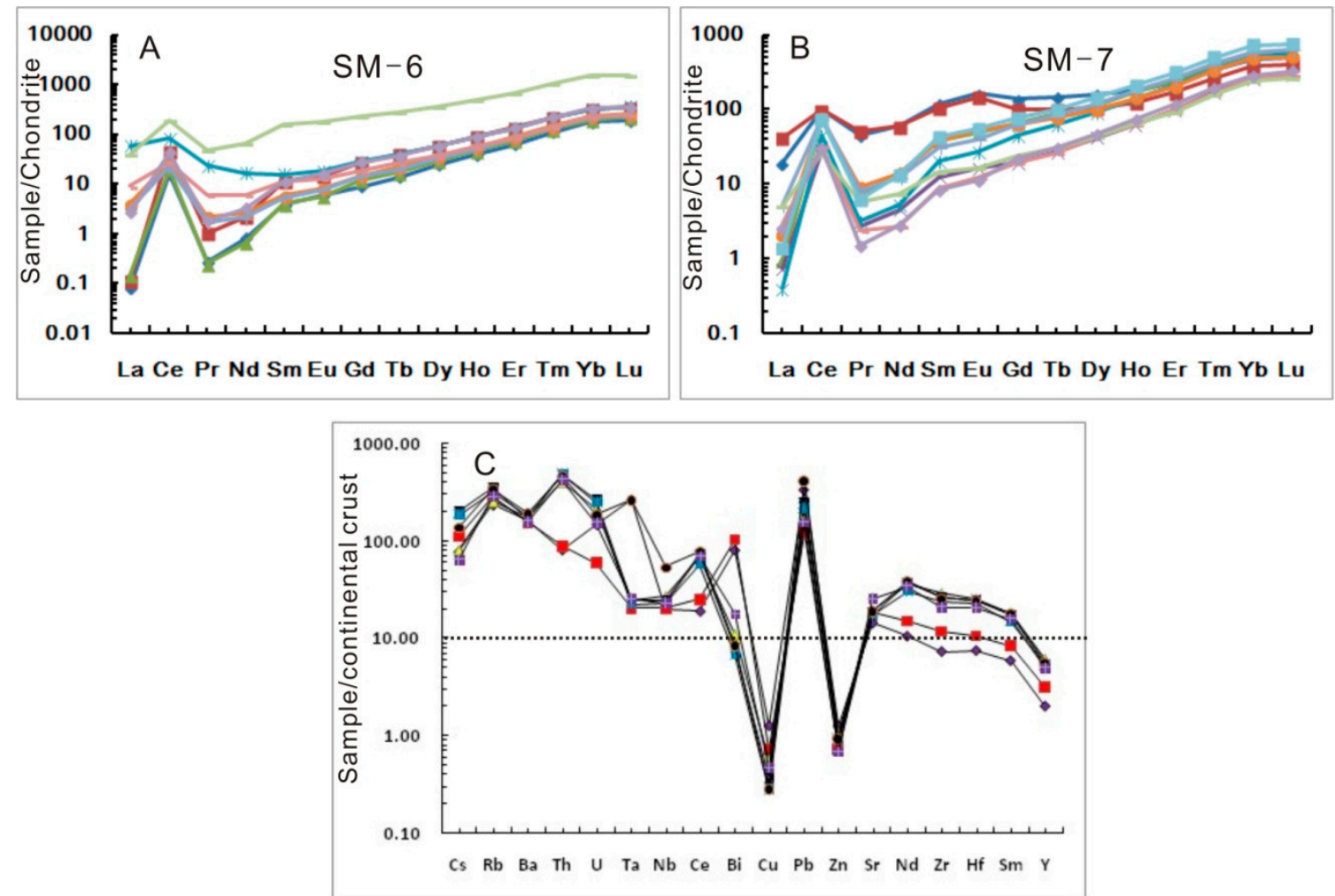

Figure 9. Chondrite-and average continental crust normalized REE distribution diagrams for different zircons from the BA Sin the studied area. (A,B) Chondritenormalized REE distribution. (C) Average continental crust normalized REE distribution. The chondrite values are from $[33,34]$. The Northern and Southern Accretionary Orogens. The average continental crust is from Eizenhöfer et al. [32].

\subsection{Whole-Rock Major and Trace Element Geochemistry}

The BAS is silica-undersaturated with abundances of $\mathrm{SiO}_{2}$ (54.97-63.43 wt.\%) $\mathrm{TiO}_{2}$ (0.33-1.34 wt.\%), $\mathrm{Al}_{2} \mathrm{O}_{3}$ (17.14-22.14 wt.\%), $\mathrm{CaO}$ (0.05-1.01 wt.\%), $\mathrm{Na}_{2} \mathrm{O}\left(0.15-5.12\right.$ wt.\%), $\mathrm{K}_{2} \mathrm{O}$ (7.23-14.18 wt.\%), $\mathrm{FeO}_{\mathrm{T}}\left(2.65-6.18\right.$ wt.\%), $\mathrm{MgO}\left(0.22-0.75\right.$ wt.\%), $\mathrm{Mg}^{\#}$ (11-29) and total alkalis $\left(\mathrm{Na}_{2} \mathrm{O}+\mathrm{K}_{2} \mathrm{O}=13.23-14.42 \mathrm{wt} . \%\right)$ (Table 3). In the selected elements vs. $\mathrm{MgO}$ diagrams (Figure 10), all syenites display regular trends of decreasing $\mathrm{Fe}_{2} \mathrm{O}_{3}, \mathrm{CaO}, \mathrm{TiO}_{2}, \mathrm{P}_{2} \mathrm{O}_{5}$, and increasing $\mathrm{SiO}_{2}$ and $\mathrm{Al}_{2} \mathrm{O}_{3}$ with decreasing $\mathrm{MgO}$. They are high- $\mathrm{K}$ rocks with $\mathrm{Na}_{2} \mathrm{O} / \mathrm{K}_{2} \mathrm{O}$ ratios less than 1 . In the chondrite-normalized REE diagram, they are strongly enriched in LREE relative to HREE, with $(\mathrm{La} / \mathrm{Yb})_{\mathrm{N}}$ and $(\mathrm{Gd} / \mathrm{Yb})_{\mathrm{N}}$ ratios of 28.18-147.09 and 2.60-8.20 and negative Eu anomalies $\left(\mathrm{Eu} / \mathrm{Eu}^{*}\right)_{\mathrm{N}}$ $=0.61-0.85$. The alkaline syenites are enriched in LILE (e.g., Rb, Th), LREEs, $\mathrm{Zr}$ and Hf and have moderate negative anomalies in $\mathrm{Ba}, \mathrm{Nb}$, $\mathrm{Ta}$ and $\mathrm{Sr}$ (Table 4). 

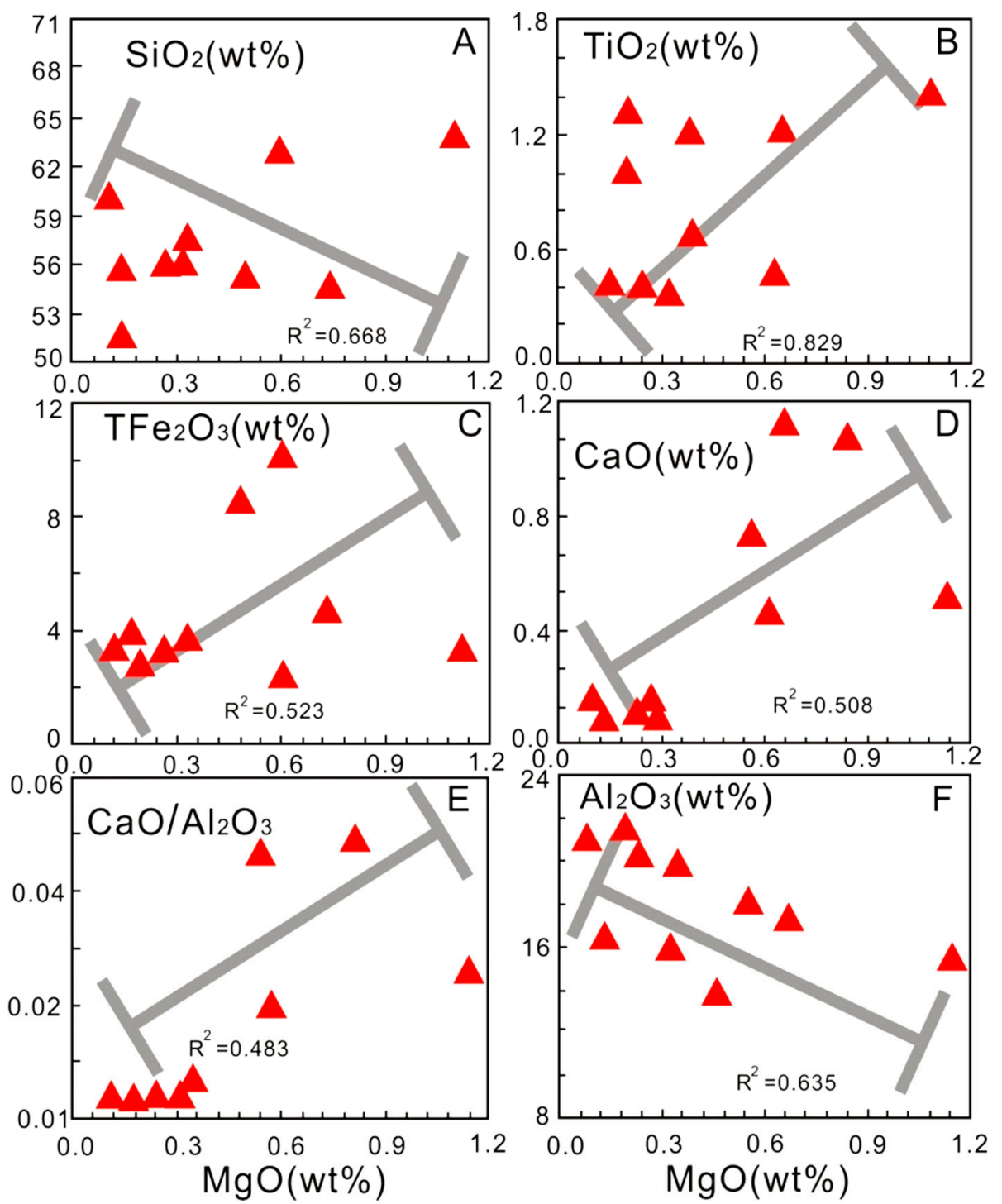

Figure 10. Selected elements vs. $\mathrm{MgO}$ diagrams (A) $\mathrm{SiO}_{2}$, (B) $\mathrm{TiO}_{2}$, (C) $\mathrm{TFe}_{2} \mathrm{O}_{3}$, (D) $\mathrm{CaO}$, (E) $\mathrm{CaO} / \mathrm{Al}_{2} \mathrm{O}_{3}$, (F) $\mathrm{Al}_{2} \mathrm{O}_{3}$. Note: red $\Delta$ represents syenite samples. 
Table 3. Zircon trace elements for samplesBLC-1 and BLC-2 ( $\mu \mathrm{g} / \mathrm{g})$.

\begin{tabular}{|c|c|c|c|c|c|c|c|c|c|c|c|c|c|c|c|c|c|c|c|c|c|}
\hline $\begin{array}{c}\text { BLC-1 } \\
\text { Plot }\end{array}$ & $\mathrm{Ti}$ & $\mathbf{L a}$ & $\mathrm{Ce}$ & Pr & $\mathrm{Nd}$ & Sm & $\mathbf{E u}$ & Gd & $\mathbf{T b}$ & Dy & Ho & Er & $\mathrm{Tm}$ & $\mathrm{Yb}$ & Lu & $\Sigma$ REE & LREE & HREE & $\begin{array}{l}\text { LREE/ } \\
\text { HREE }\end{array}$ & $\delta \mathbf{E u}$ & $\delta \mathrm{Ce}$ \\
\hline 1 & 14.3 & 7.0 & 94.6 & 6.3 & 67.2 & 7.7 & 14.2 & 42.3 & 8.6 & 60.9 & 15.5 & 57.4 & 11.9 & 118.1 & 18.7 & 525.3 & 191.9 & 333.4 & 0.6 & 1.3 & 3.1 \\
\hline 2 & 16.4 & 15.3 & 95.0 & 7.0 & 69.3 & 6.4 & 12.7 & 31.1 & 5.9 & 39.9 & 10.7 & 42.6 & 9.3 & 95.5 & 15.2 & 446.8 & 196.7 & 250.0 & 0.8 & 1.4 & 2.1 \\
\hline 3 & 5.5 & 0.3 & 71.8 & 1.0 & 70.0 & 8.6 & 4.3 & 20.5 & 5.0 & 43.7 & 14.4 & 61.0 & 14.3 & 143.1 & 23.2 & 421.1 & 96.0 & 325.1 & 0.3 & 0.9 & 18.7 \\
\hline 4 & 4.2 & 0.3 & 27.0 & 0.4 & 63.2 & 6.9 & 1.4 & 5.9 & 1.6 & 16.0 & 5.4 & 44.7 & 6.1 & 64.6 & 11.0 & 170.4 & 35.1 & 135.3 & 0.3 & 1.0 & 16.4 \\
\hline 5 & 4.6 & 0.2 & 43.9 & 0.4 & 73.8 & 5.8 & 2.4 & 14.1 & 3.7 & 35.7 & 12.0 & 52.7 & 12.4 & 126.5 & 21.4 & 333.7 & 55.4 & 278.3 & 0.2 & 0.8 & 25.4 \\
\hline 6 & 7.3 & 0.8 & 71.8 & 1.3 & 69.9 & 8.7 & 4.7 & 19.1 & 4.4 & 37.3 & 12.1 & 50.0 & 11.8 & 121.2 & 18.9 & 371.8 & 97.1 & 274.7 & 0.4 & 1.1 & 13.4 \\
\hline 7 & 5.0 & 1.9 & 73.8 & 1.1 & 68.8 & 7.3 & 3.4 & 18.9 & 4.9 & 45.4 & 15.0 & 64.8 & 14.6 & 146.7 & 24.5 & 431.1 & 96.3 & 334.8 & 0.3 & 0.9 & 11.6 \\
\hline 8 & 4.4 & 1.1 & 30.5 & 0.3 & 71.9 & 6.1 & 1.1 & 5.9 & 1.5 & 16.3 & 5.5 & 64.9 & 6.0 & 64.5 & 11.0 & 172.4 & 36.9 & 135.5 & 0.3 & 0.9 & 12.0 \\
\hline 9 & 4.9 & 1.8 & 24.8 & 0.8 & 65.2 & 7.3 & 1.4 & 7.3 & 1.8 & 16.3 & 5.6 & 53.2 & 5.6 & 59.3 & 10.0 & 166.3 & 37.4 & 128.9 & 0.3 & 0.9 & 4.8 \\
\hline 10 & 5.3 & 0.9 & 29.0 & 0.2 & 72.0 & 8.0 & 1.0 & 6.8 & 1.8 & 17.5 & 6.4 & 59.5 & 7.0 & 71.7 & 12.5 & 188.2 & 35.0 & 153.1 & 0.2 & 0.7 & 14.9 \\
\hline 11 & 5.7 & 0.5 & 70.7 & 0.8 & 69.6 & 9.8 & 4.8 & 24.0 & 5.8 & 55.7 & 18.3 & 78.6 & 17.8 & 181.3 & 28.9 & 506.6 & 96.3 & 410.3 & 0.2 & 0.9 & 19.7 \\
\hline 12 & 9.9 & 49.4 & 79.4 & 13.3 & 65.9 & 8.7 & 15.8 & 47.6 & 9.9 & 77.0 & 21.4 & 85.2 & 18.9 & 185.5 & 30.5 & 828.5 & 352.5 & 476.0 & 0.7 & 1.3 & 1.6 \\
\hline 13 & 5.8 & 0.1 & 52.1 & 0.2 & 72.9 & 8.4 & 1.8 & 11.5 & 3.0 & 31.6 & 11.4 & 52.1 & 12.0 & 125.5 & 21.6 & 328.7 & 60.1 & 268.6 & 0.2 & 0.8 & 60.1 \\
\hline 14 & 7.4 & 5.4 & 37.3 & 0.8 & 74.6 & 6.5 & 1.3 & 6.0 & 1.8 & 17.9 & 6.2 & 58.7 & 6.9 & 74.7 & 12.5 & 206.4 & 51.7 & 154.6 & 0.3 & 1.0 & 3.8 \\
\hline 15 & 8.9 & 0.6 & 96.3 & 1.0 & 70.6 & 6.7 & 6.5 & 27.0 & 6.7 & 61.0 & 19.6 & 84.7 & 19.2 & 195.7 & 31.8 & 572.3 & 126.6 & 445.7 & 0.3 & 1.1 & 23.8 \\
\hline 16 & 3.8 & 2.5 & 65.9 & 2.0 & 69.8 & 4.5 & 1.6 & 7.5 & 1.7 & 16.5 & 5.4 & 24.2 & 5.7 & 60.3 & 10.8 & 218 & 86 & 132 & 0.7 & 0.8 & 6.4 \\
\hline 17 & 3.4 & 0.1 & 20.2 & 0.1 & 70.8 & 6.9 & 0.6 & 3.7 & 1.0 & 10.9 & 3.9 & 18.5 & 4.4 & 47.6 & 8.8 & 121 & 23 & 99 & 0.2 & 0.8 & 59.7 \\
\hline 18 & 3.7 & 0.0 & 10.8 & 0.0 & 70.2 & 7.3 & 0.2 & 1.2 & 0.4 & 4.0 & 1.5 & 7.4 & 1.8 & 20.2 & 3.7 & 52 & 11 & 40 & 0.3 & 0.7 & 102.1 \\
\hline 19 & 5.1 & 0.0 & 23.8 & 0.1 & 68.9 & 6.4 & 0.8 & 4.7 & 1.3 & 13.3 & 4.9 & 22.7 & 5.2 & 58.3 & 10.0 & 147 & 27 & 120 & 0.2 & 0.8 & 77.9 \\
\hline 20 & 4.1 & 0.0 & 26.3 & 0.1 & 71.0 & 7.4 & 0.8 & 5.3 & 1.4 & 15.6 & 5.5 & 25.7 & 6.1 & 66.0 & 11.6 & 167 & 30 & 137 & 0.2 & 0.8 & 90.3 \\
\hline 21 & 4.0 & 0.1 & 30.7 & 0.5 & 66.0 & 6.7 & 3.4 & 19.7 & 5.2 & 49.9 & 16.9 & 74.2 & 17.1 & 76.4 & 29.7 & 436 & 47 & 389 & 0.1 & 0.8 & 17.1 \\
\hline 22 & 4.7 & 0.0 & 24.8 & 0.1 & 81.0 & 5.4 & 0.7 & 5.0 & 1.3 & 14.1 & 5.0 & 22.9 & 5.7 & 61.5 & 10.3 & 154 & 28 & 126 & 0.2 & 0.8 & 96.5 \\
\hline 23 & 3.3 & 1.9 & 32.3 & 0.4 & 62.3 & 5.9 & 1.1 & 7.2 & 2.0 & 20.0 & 7.1 & 31.4 & 7.5 & 77.6 & 13.8 & 206 & 40 & 167 & 0.2 & 0.8 & 8.8 \\
\hline 24 & 5.0 & 6.4 & 40.9 & 1.2 & 74.1 & 1.7 & 0.9 & 5.1 & 1.4 & 14.3 & 5.0 & 22.6 & 5.2 & 56.0 & 9.9 & 175 & 55 & 119 & 0.5 & 0.8 & 3.2 \\
\hline 25 & 5.4 & 0.7 & 17.7 & 0.2 & 61.3 & 6.7 & 0.5 & 2.9 & 0.8 & 8.7 & 3.1 & 14.8 & 3.7 & 39.8 & 7.1 & 102 & 21 & 81 & 0.3 & 0.7 & 11.5 \\
\hline 26 & 3.9 & 0.9 & 26.1 & 0.4 & 72.8 & 7.2 & 1.2 & 4.8 & 1.4 & 12.8 & 4.6 & 20.8 & 5.0 & 53.2 & 9.7 & 146 & 33 & 112 & 0.3 & 1.1 & 10.3 \\
\hline 27 & 4.7 & 0.1 & 12.0 & 0.1 & 80.4 & 7.4 & 0.3 & 1.9 & 0.6 & 5.9 & 2.5 & 11.6 & 3.1 & 33.8 & 6.1 & 79 & 13 & 65 & 0.2 & 0.9 & 40.3 \\
\hline 28 & 6.6 & 0.0 & 29.7 & 0.1 & 61.6 & 7.6 & 1.0 & 6.2 & 1.7 & 16.8 & 6.1 & 26.9 & 6.5 & 70.3 & 11.8 & 180 & 34 & 146 & 0.2 & 0.9 & 69.2 \\
\hline
\end{tabular}


Table 4. Whole rock analyses of the major (wt.\%) and trace (ppm) elemental compositions of the BAS.

\begin{tabular}{|c|c|c|c|c|c|c|c|c|c|c|}
\hline \multirow{2}{*}{$\begin{array}{c}\text { Lithology } \\
\text { Number }\end{array}$} & \multicolumn{10}{|c|}{ Alkaline Syenite } \\
\hline & BCL-5 & BCL-6 & BCL-7 & BCL-8 & BCL-9 & BCL-10 & BCL-11 & BCL-12 & BCL-13 & BCL-14 \\
\hline $\mathrm{SiO}_{2}$ & 57.12 & 63.43 & 57.68 & 56.93 & 54.97 & 55.87 & 65.82 & 61.19 & 57.63 & 50.67 \\
\hline $\mathrm{Al}_{2} \mathrm{O}_{3}$ & 22.14 & 18.48 & 20.85 & 21.66 & 17.14 & 13.30 & 16.73 & 17.98 & 17.57 & 21.18 \\
\hline $\mathrm{Fe}_{2} \mathrm{O}_{3}$ & 2.93 & 2.04 & 4.01 & 3.55 & 5.50 & 11.21 & 0.96 & 4.03 & 8.81 & 4.41 \\
\hline $\mathrm{MgO}$ & 0.22 & 0.62 & 0.32 & 0.25 & 0.75 & 0.45 & 1.18 & 0.13 & 0.29 & 0.17 \\
\hline $\mathrm{Mg}^{\#}$ & 12 & 29 & 13 & 11 & 17 & 7 & 51 & 6 & 7 & 9 \\
\hline $\mathrm{CaO}$ & 0.07 & 0.52 & 0.05 & 0.05 & 1.01 & 0.75 & 0.43 & 0.13 & 0.12 & 1.35 \\
\hline $\mathrm{Na}_{2} \mathrm{O}$ & 0.25 & 5.12 & 0.15 & 0.36 & 4.56 & 7.52 & 4.79 & 2.85 & 0.68 & 10.55 \\
\hline $\mathrm{K}_{2} \mathrm{O}$ & 14.18 & 7.23 & 14.13 & 13.83 & 8.67 & 6.50 & 6.38 & 10.54 & 10.39 & 3.51 \\
\hline $\mathrm{MnO}$ & 0.07 & 0.04 & 0.04 & 0.05 & 0.18 & 0.11 & 0.08 & 0.08 & 0.18 & 0.29 \\
\hline $\mathrm{TiO}_{2}$ & 0.33 & 0.46 & 0.44 & 0.44 & 1.34 & 0.72 & 1.33 & 1.18 & 1.15 & 0.97 \\
\hline $\mathrm{P}_{2} \mathrm{O}_{5}$ & 0.09 & 0.19 & 0.05 & 0.10 & 0.05 & 0.02 & 0.14 & 0.14 & 0.05 & 0.02 \\
\hline LOI & 2.42 & 1.06 & 1.90 & 2.41 & 4.59 & 2.33 & 1.03 & 1.59 & 2.93 & 6.23 \\
\hline $\mathrm{FeO}$ & 0.19 & 0.81 & 0.38 & 0.36 & 1.23 & 1.20 & 1.12 & 0.15 & 0.21 & 0.64 \\
\hline $\mathrm{F}$ & 0.00 & 0.00 & 0.00 & 0.00 & 0.00 & 0.00 & 0.00 & 0.00 & 0.00 & 0.00 \\
\hline Total & 99.99 & 99.99 & 99.99 & 99.99 & 99.98 & 99.98 & 99.99 & 99.99 & 99.99 & 99.98 \\
\hline $\mathrm{A} / \mathrm{CNK}$ & 1.39 & 1.07 & 1.33 & 1.38 & 0.91 & 0.64 & 1.07 & 1.10 & 1.39 & 0.90 \\
\hline A/NK & 1.40 & 1.14 & 1.34 & 1.39 & 1.01 & 0.68 & 1.13 & 1.12 & 1.42 & 1.00 \\
\hline $\mathrm{AV}$ & 0.65 & 0.67 & 0.68 & 0.66 & 0.77 & 1.05 & 0.67 & 0.74 & 0.63 & 0.66 \\
\hline $\mathrm{AR}$ & 4.71 & 4.71 & 5.31 & 4.77 & 6.37 & 934.33 & 4.72 & 6.67 & 4.34 & 4.32 \\
\hline FL & 99.52 & 95.95 & 99.63 & 99.63 & 92.89 & 94.92 & 96.27 & 99.03 & 98.95 & 91.22 \\
\hline MF & 93.38 & 82.19 & 93.16 & 93.99 & 90.04 & 96.47 & 63.74 & 97.10 & 96.91 & 96.77 \\
\hline OX & 0.06 & 0.28 & 0.09 & 0.09 & 0.18 & 0.10 & 0.54 & 0.04 & 0.02 & 0.13 \\
\hline$\delta$ & 14.74 & 7.46 & 13.89 & 14.46 & 14.62 & 15.27 & 5.46 & 9.85 & 8.37 & 25.77 \\
\hline $\mathrm{Li}$ & 127 & 11.4 & 123 & 77.4 & 104 & 88.3 & 13.3 & 10.2 & 71.2 & 39.9 \\
\hline $\mathrm{Be}$ & 18.2 & 4.8 & 10.6 & 36.6 & 32.8 & 12.8 & 6.35 & 4.11 & 14.5 & 69 \\
\hline $\mathrm{Sc}$ & 7.96 & 15.2 & 3.88 & 2.9 & 21.3 & 17.1 & 6.99 & 4.64 & 7.48 & 15.7 \\
\hline $\mathrm{V}$ & 49.4 & 51.2 & 44.4 & 79.8 & 111 & 235 & 37.1 & 86.9 & 151 & 56.3 \\
\hline $\mathrm{Cr}$ & 7.35 & 11.1 & 3.37 & 4.06 & 1.94 & 0.604 & 19.2 & 3.78 & 1.07 & 1.88 \\
\hline Co & 6.94 & 5.25 & 5.4 & 10.2 & 7.27 & 2.78 & 6.07 & 6.15 & 6.46 & 3.72 \\
\hline $\mathrm{Ni}$ & 3.99 & 6.65 & 3.42 & 4.1 & 1.46 & 0.12 & 12.8 & 3.14 & 0.816 & 0.777 \\
\hline $\mathrm{Cu}$ & 36.6 & 7.94 & 22.9 & 41.1 & 3.65 & 1.19 & 1.95 & 31.4 & 6.3 & 2.57 \\
\hline $\mathrm{Zn}$ & 152 & 65.2 & 74 & 172 & 162 & 113 & 45.3 & 104 & 78.8 & 135 \\
\hline $\mathrm{Ga}$ & 34.6 & 27 & 36 & 45.1 & 42.4 & 53.9 & 26 & 31.8 & 50.6 & 63.1 \\
\hline $\mathrm{Rb}$ & 110 & 121 & 100 & 144 & 135 & 104 & 106 & 94 & 101 & 106 \\
\hline $\mathrm{Sr}$ & 1083 & 1000 & 1026 & 1207 & 1621 & 1140 & 977 & 971 & 1040 & 1003 \\
\hline $\mathrm{Y}$ & 26 & 17.5 & 23.3 & 18.2 & 29.1 & 6.77 & 34 & 18.8 & 202 & 255 \\
\hline $\mathrm{Nb}$ & 34.2 & 55.9 & 35.6 & 27.1 & 96.9 & 69.2 & 95.6 & 59.5 & 276 & 325 \\
\hline Mo & 0.682 & 1.62 & 0.71 & 0.824 & 0.388 & 0.145 & 1.3 & 1.98 & 0.642 & 0.336 \\
\hline $\mathrm{Cd}$ & 0.186 & 0.034 & 0.091 & 0.119 & 0.175 & 0.191 & 0.098 & 0.225 & 0.82 & 1.24 \\
\hline In & 0.013 & 0.03 & 0.023 & 0.035 & 0.043 & 0.057 & 0.017 & 0.035 & 0.081 & 0.028 \\
\hline $\mathrm{Sb}$ & 0.331 & 0.106 & 0.434 & 0.426 & 0.13 & 0.062 & 0.096 & 1.01 & 12.8 & 0.1 \\
\hline Cs & 1.51 & 1.36 & 2.65 & 5.57 & 1.52 & 3.74 & 1.18 & 1.52 & 6.53 & 0.792 \\
\hline $\mathrm{Ba}$ & 2188 & 1516 & 3094 & 3486 & 1085 & 212 & 2375 & 645 & 902 & 273 \\
\hline $\mathrm{La}$ & 174 & 179 & 71.5 & 127 & 365 & 59.5 & 180 & 168 & 972 & 319 \\
\hline $\mathrm{Ce}$ & 129 & 184 & 159 & 127 & 116 & 96.3 & 174 & 129 & 124 & 174 \\
\hline $\operatorname{Pr}$ & 22.7 & 30.5 & 10.3 & 12.1 & 53.2 & 7.72 & 47.6 & 41 & 117 & 57.6 \\
\hline $\mathrm{Nd}$ & 41.6 & 49.3 & 44.9 & 49 & 37 & 40.5 & 48.2 & 51.3 & 39 & 46 \\
\hline $\mathrm{Sm}$ & 6.21 & 7.89 & 6.72 & 5.27 & 9.9 & 9.7 & 9.4 & 8.5 & 8.7 & 6.9 \\
\hline $\mathrm{Eu}$ & 3.26 & 2.65 & 1.77 & 1.04 & 4.93 & 0.355 & 5.07 & 6.62 & 15.9 & 15.6 \\
\hline $\mathrm{Gd}$ & 10.1 & 10.5 & 5.83 & 5.01 & 18 & 2.27 & 19.9 & 17.7 & 54.6 & 41.1 \\
\hline $\mathrm{Tb}$ & 1.36 & 1.23 & 0.909 & 0.735 & 1.91 & 0.228 & 2.73 & 2.07 & 7.9 & 8.09 \\
\hline Dy & 4.97 & 3.89 & 3.98 & 3.44 & 5.95 & 0.916 & 9.04 & 5.47 & 34.6 & 44 \\
\hline Ho & 0.845 & 0.615 & 0.693 & 0.579 & 0.902 & 0.193 & 1.34 & 0.738 & 6.24 & 8.71 \\
\hline $\mathrm{Er}$ & 2.59 & 2.07 & 1.95 & 1.54 & 2.85 & 0.738 & 4.2 & 2.46 & 18.3 & 25.1 \\
\hline $\mathrm{Tm}$ & 0.346 & 0.241 & 0.305 & 0.22 & 0.3 & 0.151 & 0.462 & 0.218 & 2.64 & 4.24 \\
\hline $\mathrm{Yb}$ & 2.24 & 1.43 & 1.82 & 1.36 & 1.78 & 1.22 & 2.59 & 1.27 & 15.9 & 26.2 \\
\hline $\mathrm{Lu}$ & 0.307 & 0.193 & 0.246 & 0.182 & 0.257 & 0.227 & 0.312 & 0.14 & 2.06 & 3.47 \\
\hline $\mathrm{Ta}$ & 1 & 2.93 & 0.894 & 0.5 & 4.01 & 3.87 & 7.24 & 5.77 & 8.08 & 13.2 \\
\hline W & 1.83 & 2.48 & 2.84 & 2.59 & 0.586 & 0.897 & 3.9 & 10.3 & 5.91 & 11.4 \\
\hline $\operatorname{Re}$ & 0.003 & 0.002 & 0.003 & 0.002 & 0.002 & 0.002 & 0.003 & 0.002 & 0.024 & 0.035 \\
\hline $\mathrm{Tl}$ & 1.84 & 1 & 1.53 & 1.72 & 1.86 & 1.1 & 0.82 & 1.6 & 2.72 & 2.15 \\
\hline $\mathrm{Pb}$ & 64.8 & 64.3 & 59.8 & 220 & 200 & 663 & 34.5 & 78.9 & 233 & 843 \\
\hline $\mathrm{Bi}$ & 0.069 & 0.21 & 0.076 & 0.144 & 0.456 & 0.194 & 0.177 & 0.188 & 1.03 & 0.144 \\
\hline
\end{tabular}


Table 4. Cont.

\begin{tabular}{|c|c|c|c|c|c|c|c|c|c|c|}
\hline $\begin{array}{c}\text { Lithology } \\
\text { Number }\end{array}$ & \multicolumn{10}{|c|}{ Alkaline Syenite } \\
\hline Th & 29.9 & 24.8 & 73 & 46.2 & 262 & 16.5 & 35.1 & 18.5 & 300 & 19 \\
\hline $\mathrm{Zr}$ & 1367 & 259 & 914 & 692 & 1572 & 2289 & 192 & 269 & $>5000$ & $>5000$ \\
\hline Hf & 27.1 & 8.03 & 18.9 & 18.9 & 41.2 & 57.8 & 7.41 & 8.74 & 141 & 264 \\
\hline$\Sigma \mathrm{REE}$ & 445.92 & 628.6 & 299.9 & 324.4 & 1258.9 & 192.05 & 958.24 & 856.19 & 3196.8 & 1387.0 \\
\hline HREE & 22.76 & 20.17 & 15.73 & 13.07 & 31.95 & 5.94 & 40.57 & 30.07 & 142.24 & 160.91 \\
\hline LREE/HREE & 18.59 & 30.17 & 18.06 & 23.83 & 38.41 & 31.31 & 22.62 & 27.48 & 21.47 & 7.62 \\
\hline $\mathrm{LaN} / \mathrm{YbN}$ & 55.72 & 89.79 & 28.18 & 66.98 & 147.09 & 34.98 & 49.85 & 94.89 & 43.85 & 8.73 \\
\hline$(\mathrm{La} / \mathrm{Sm}) \mathrm{N}$ & 8.69 & 8.67 & 6.70 & 15.17 & 10.99 & 21.65 & 3.91 & 4.15 & 10.42 & 4.28 \\
\hline$(\mathrm{Gd} / \mathrm{Yb}) \mathrm{N}$ & 3.65 & 5.95 & 2.60 & 2.99 & 8.20 & 1.51 & 6.23 & 11.30 & 2.78 & 1.27 \\
\hline$\delta \mathrm{Eu}$ & 0.85 & 0.67 & 0.84 & 0.61 & 0.76 & 0.55 & 0.61 & 0.90 & 0.84 & 1.06 \\
\hline
\end{tabular}

Note: FelsicIndex $(\mathrm{FL})=100 \times\left(\mathrm{Na}_{2} \mathrm{O}+\mathrm{K}_{2} \mathrm{O}\right) /\left(\mathrm{CaO}+\mathrm{Na}_{2} \mathrm{O}+\mathrm{K}_{2} \mathrm{O}\right)($ wt. $\%) ;$ Mafic Index $(\mathrm{MF})=100 \times\left(\mathrm{Fe}_{2} \mathrm{O}_{3}\right.$

$+\mathrm{FeO}) /\left(\mathrm{MgO}+\mathrm{Fe}_{2} \mathrm{O}_{3}+\mathrm{FeO}\right)($ wt. $\%) ; \mathrm{A} / \mathrm{CNK}=\mathrm{Al}_{2} \mathrm{O}_{3} /\left(\mathrm{CaO}+\mathrm{Na}_{2} \mathrm{O}+\mathrm{K}_{2} \mathrm{O}\right) ; \mathrm{A} / \mathrm{NK}=\mathrm{Al}_{2} \mathrm{O}_{3} /\left(\mathrm{Na}_{2} \mathrm{O}+\mathrm{K}_{2} \mathrm{O}\right) ;$ $\mathrm{AR}=\left[\mathrm{Al}_{2} \mathrm{O}_{3}+\mathrm{CaO}+\left(\mathrm{Na}_{2} \mathrm{O}+\mathrm{K}_{2} \mathrm{O}\right)\right] /\left[\mathrm{Al}_{2} \mathrm{O}_{3}+\mathrm{CaO}-\left(\mathrm{Na}_{2} \mathrm{O}+\mathrm{K}_{2} \mathrm{O}\right)\right](\mathrm{wt} . \%) \cdot \mathrm{Mg}^{\#}=\mathrm{Mg}^{2+} /\left(\mathrm{Mg}^{2+}+\mathrm{Fe}^{2+}\right) \times 100$.

\section{Discussion}

\subsection{Zircon Crystallization Condition}

Defining the conditions of zircon crystallization in magmatic environments is one of the aspects in whole-rock geochemistry in order to understand which geological processes are being dated. Furthermore, the Ti-in-zircon thermometer has the potential to create an invaluable link between $\mathrm{U}-\mathrm{Pb}$ ages and temperatures measured in-situ in zircon (e.g., [31-33]). The Ti content of zircon coexisting with rutile or other Ti-rich phases has a strong dependence on temperature (T) [35]. The thermometer can be applied to individual grains, and they provide estimates of crystallization $\mathrm{T}$ for minerals [36,37]. However, rutile is rarely observed in our studied samples. Sphene is easily found in studied samples [38]. Ferry and Watson [39] revised the calibration of the Ti-in-zircon thermometer and made it more generally useful by extending its application to rocks devoid of quartz and, in the case of the zircon thermometer, devoid of rutile [37]. In the case of rocks that lack quartz and/or rutile, and for which values of $\alpha_{\mathrm{SiO} 2}$ (the activity of $\mathrm{SiO}_{2}$ ) and $\alpha_{\mathrm{TiO} 2}$ (the activity of $\mathrm{TiO}_{2}$ ) may be independently estimated from mineral equilibria, accurate estimates of $\mathrm{T}$ can still be made from equation:

$$
\log (\text { ppm Ti-in-zircon })=(5.711 \pm 0.072)-(4800 \pm 86) / \mathrm{T}(\mathrm{K})-\log \alpha \mathrm{SiO}_{2}+\log \alpha_{\mathrm{TiO} 2}
$$

Values of $\alpha_{\mathrm{SiO} 2}$ in silicic melts at appropriate magmatic Ts are rarely below 0.5 and typically $0.6-0.9$ [35-37]. Zircons with Ti contents that record $\mathrm{T}=750{ }^{\circ} \mathrm{C}$ under conditions of $\alpha_{\mathrm{SiO} 2}=1$ record $\mathrm{T}=820{ }^{\circ} \mathrm{C}$ for $\alpha_{\mathrm{SiO} 2}=0.5$. The uncommon occurrence of nepheline indicate that $\alpha_{\mathrm{SiO} 2}$ in most crustal rocks is not usually less than $\approx 0.5$ (calculated from Berman's 1988 database). Therefore, we have applied the model of Ferry and Watson [39] to identify $\mathrm{Zr}$ crystallization temperatures in the BAS [37]. The studied samples (BLC-1 and BLC-2) have the calculated magmatic crystallization temperature average values of $766^{\circ} \mathrm{C}$ and $816^{\circ} \mathrm{C}$, respectively.

\subsection{Geochronology of Magmatismandgeological Implications}

Previous dating of the Bailinchuan alkaline complex was mainly conducted by whole rock $\mathrm{Rb}$-Srisochron, $\mathrm{K}-\mathrm{Ar}, \mathrm{U}-\mathrm{Pb}$ and ${ }^{40} \mathrm{Ar} /{ }^{39} \mathrm{Ar}$ methods, and gave a wide range of ages between 197 and $241 \mathrm{Ma}[9,13,14,26]$. U-Pb zircon LA-ICP-MS dating presented highly accordant age (226-229 Ma) for twosyenites samples in Bailinchuan district in this study. The age presented here is analogous to previous research. All those ages obtained in this and previous investigations support that there is an episode of Late Triassic magmatism in the district. 
Sometimes syenite dating has been susceptible to interference because there are insufficient protogenetic magmatic zircons. So it should be determined whether the zircons we analyzed are all inherited or xenolith zircons. Inherited grains should be (1) broadly similar to grains in the host rocks, the likely inheritance sources for small volume, locally-derived granitic rocks, and (2) similar between samples. The observed zircon morphology displayed none of the above features. Therefore, those Middle-Late Triassic zircons are not inherited from the host rock or wall rock. Zircons from the four syenite samples differ from each other which also reflect they are not inherited from one same source. More importantly, zircon grains that formed during igneous crystallization should have (1) zoning typical of grains crystallized from magma, (2) similar composition and zoning within samples, and (3) different composition and zoning between samples. Analyzed zircons satisfy all aforementioned criteria, and therefore, are interpreted to be protogenetic magmatic zircons, where the age of 226-229 Ma can be interpreted as the magma crystallization age for BAS. Previous studies showed that the Paleo-Asiaoceanic slab was subducted beneath the NCC during the period of the Paleozoic, forming the early Paleozoic arc-accretionary series with zircon U-Pb ages of 490-446 Ma [40,41] and the late Paleozoic Andean-type continental arc magmatism on the northern margin of the NCC (390-270 Ma) [42]. Subsequently, the endof the subduction could be constrained at ca. $290 \mathrm{Ma}$ or later [42,43], and voluminous post-collisional granites were formed (270-230 Ma; [44]). Eizenhöferand Zhao [45] have concluded that the closure of the east-westoriented Palaeo-Asian Ocean via bidirectional subduction in the Permian to Early Triassic.

In the Late Triassic, the east-west trending alkaline magmatic belt was emplaced on the northern NCC. The BAS in this study, located at the eastern part of the alkaline rock belt, has zircon $\mathrm{U}-\mathrm{Pb}$ age of 226-229 Ma. The Baotoudong syenites, located at the western end part of the belt, have a zircon U-Pb age of $214 \mathrm{Ma}$ [8]. In addition, the Fanshan and Yaojiazhuang ultramafic-syenitic complexes, located in the middle part of the belt, have zircon U-Pb ages of ca. $220 \mathrm{Ma}$ and $209 \pm 2.1 \mathrm{Ma}$, respectively [8]; the Liangcheng syenites, located in the middle part of the belt, have a zircon LA-ICP-MS U-Pb age of 227.7 Ma [8]. The results of the U-Pb analyses of zircons from published data suggested that the timing of these magmatic activities should be limited to the late Triassic. A combination of the results of the current research about the late Triassic alkaline rock belt in the northern NCC [8,13], its suggested that these alkaline intrusions were simultaneously emplaced at around $220 \mathrm{Ma}$, in the Late Triassic In addition, these Triassic intrusions have a lot in common: (1) they were emplaced on the northern NCC with respect to spatial distribution; (2) they are potassic-ultrapotassic; (3) the emplacement age of these alkaline rocks are concentrated around the Late Triassic (Figure 11).

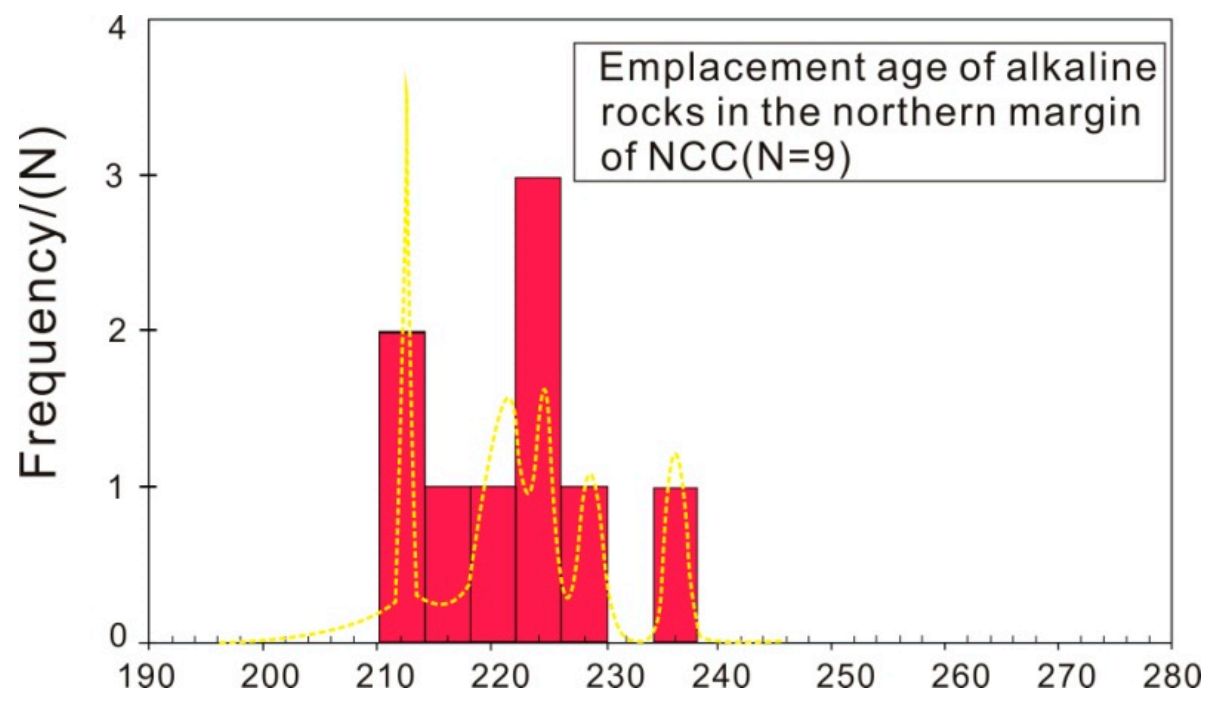

Figure 11. Histogram of alkaline rock emplacement ages in the northern margin of NCC. Data sources: The alkaline rocks are from $[8,13,45]$ and this study. 


\subsection{Magma Sourcefor BAS}

The BAS have highlight rare earth element (LREE) contents and high abundances of large-ion lithophile elements (LILE) with $\mathrm{Rb}=141-444 \mathrm{ppm}, \mathrm{Sr}=140-5000 \mathrm{ppm}$ and $\mathrm{Ba}=212-3486 \mathrm{ppm}$, which are much higher than that of crustal rocks (average $\mathrm{Rb}$, $\mathrm{Sr}$ and Ba contents of crustal rocks is 49,320 and $456 \mathrm{ppm}$, respectively) [46], indicating that the mantle source for the BAS is enriched in LREE and LILE. The BAS has relatively low $\mathrm{MgO}(0.13-1.18 \mathrm{wt} . \%), \mathrm{Mg}^{\#}(6-51)$ and intermediate $\mathrm{SiO}_{2}$ contents (Table 3), suggesting that they formed from evolved derivates of a mantle-derived magma. The enriched lithospheric mantle mainly contains olivine, orthopyroxene, and clinopyroxene, with minor amounts of amphibole, phlogopite, apatite, carbonate, ilmenite, rutile and a number of exotic oxides known from metasomatized mantle xenoliths (e.g., [47,48]). The primitive magma of the BAS should have relatively low $\mathrm{Ni}$ in comparison with tholeiitic basalts, suggesting that olivine and orthopyroxene constituted a smaller part of the melting assemblage than in an ordinary lherzolite, consistent with a metasomatized source for the Bailinchuan rocks. The evolution of clinopyroxene from biotite-rich to aegirine-rich is typical of alkaline complexes worldwide [1,43]. Clinopyroxenes in metasomatized mantle xenoliths may contain from 100-600 ppm Sr and 10-100 ppm La (e.g., [49]) and must thus contain a significant proportion of the Sr and REE budget of the melt. Melting of clinopyroxene has been invoked to explain the high $\mathrm{CaO}$ and $\mathrm{CaO} / \mathrm{Al}_{2} \mathrm{O}_{3}$ of such mantle-derived magmas [50,51]. However, elements such as Ba do not exceed 1-2 ppm in clinopyroxene, which even at $1 \%$ melting will not yield the large degrees of enrichment seen in the BAS.

The BAS, with high $\mathrm{K}_{2} \mathrm{O}$ concentrations (3.51-14.18 wt.\%), indicate a K-bearing phase, such as amphibole or phlogopite in their source. Melts in equilibrium with phlogopite are expected to have higher $\mathrm{Rb} / \mathrm{Sr}(>0.1)$ and lower $\mathrm{Ba} / \mathrm{Rb}(<20)$ ratios than those from an amphibole-bearing sources [52]. In addition, assuming that all $\mathrm{K}$ in the source is in phlogopite (or $\mathrm{K}$-amphibole; contributions by all other mantle phases are considered to be trivial), the concentrations of $\mathrm{K}$ in the melt is dependent only on the proportion in which phlogopite (or K-amphibole) enters the melt and will remain constant as long as residual phlogopite (or K-amphibole) remains. Using a $\mathrm{K}_{2} \mathrm{O}$ content of $9.8 \%$ for typical mantle phlogopite, the concentration of $\mathrm{K}$ in an equilibrium partial melt will remain constant at just over 40,000 ppm ( 160 times primitive mantle); while using a $\mathrm{K}_{2} \mathrm{O}$ content of $1.7 \%$ for mantle amphibole, the concentration of $\mathrm{K}$ in equilibrium with partial melt will remain constant at just over 7000 ppm ( 30 times primitive mantle) [53]. The K content of the BAS is on the order of 140-500 times primitive mantle, suggesting that phlogopite was the major K-bearing phase in the source of the BAS during partial melting. Mantle apatite or metasomatic oxides (i.e., Ba-K titanates, lindsleyite and mathiasite, LIMA phases) have very high concentrations of $\mathrm{Ba}$, Sr, LREE, $\mathrm{U}$, Th and $\mathrm{Pb}$ (e.g., [49]). The apatite and LIMA phases in the melting assemblage would explain the high LREE, Ba, $\mathrm{U}$, Th and $\mathrm{Pb}$ concentrations in the BAS. Furthermore, the correlations between $\mathrm{CaO}, \mathrm{Al}_{2} \mathrm{O}_{3}, \mathrm{CaO} / \mathrm{Al}_{2} \mathrm{O}_{3}$ and $\mathrm{MgO}$ are consistent with extensive clinopyroxene and/or plagioclase fractionation (Figure 10). The clinopyroxene fractionation is also supported by the $\mathrm{V}$ and $\mathrm{Ni}$ vs. Cr diagrams (Figure 12). Clinopyroxene is an important mineral that controls the fractionation of REEs. Compared with garnet, clinopyroxene is enriched in LREEs than HREEs [54]. Thus, garnet has more influence on the depletion of heavy rare earth element (HREE). 

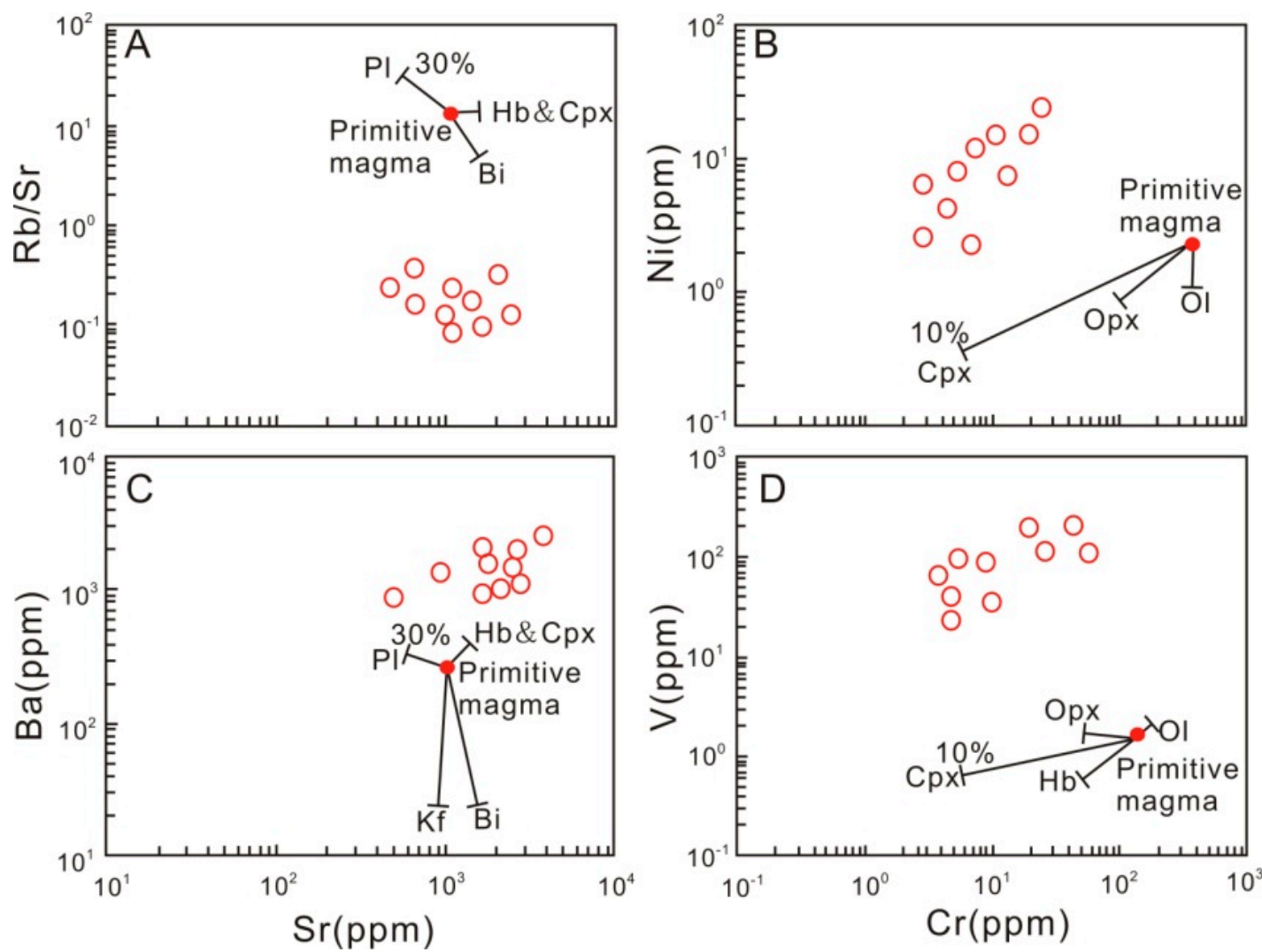

Figure 12. (A) $\mathrm{Rb} / \mathrm{Sr}$, and (C) Ba vs. Sr and (B) Ni and (D) V vs. Cr diagrams showing crystal fractionation trends. Note: hollow circlerepresents syenite samples. Solid circlerepresents intersection.

The extremely high $\left(\mathrm{La}_{\mathrm{N}} / \mathrm{Yb}_{\mathrm{N}}=8.73-147.09\right)$ and $\left(\mathrm{Gd}_{\mathrm{N}} / \mathrm{Yb}_{\mathrm{N}}=1.3-11.3\right)$ ratios, combined with low HREE abundances, suggests that garnet was a residual phase in the sources of the BAS. In addition, it has been confirmed that the partial melts of mantle source in a garnet-stable area have high $\mathrm{Dy} / \mathrm{Yb}$ ratios $(>2.5)$ and the partial melts of a mantle source in a spinel-stable area have relatively low Dy/Yb ratios $(<1.5)$ [55]. The Dy/Yb ratios range from 1.67 to 4.31 in the BAS. The mantle source of the BAS should be in the garnet-stable area $(>80 \mathrm{~km})$ as suggested by the high $\mathrm{Dy} / \mathrm{Yb}$ ratios (1.67-4.31) of the clinopyroxenites, which is consistent with the phlogopite-bearing character ( $>2 \mathrm{GPa}$, about 70-80 km). The enrichment of $\mathrm{K}$ of syenite indicate residence of high-K minerals such as phlogopite or hornblende and in $\mathrm{Dy} / \mathrm{Yb}-\mathrm{K} / \mathrm{Yb}$ plot (Figure 13), all syenite samples plot in the garnet iherzolite area. $\mathrm{Rb} / \mathrm{Sr}-\mathrm{Ba} / \mathrm{Rb}$ plot (Figure 14) was further introduced: low $\mathrm{Ba} / \mathrm{Rb}$ and $\mathrm{Rb} / \mathrm{Sr}$ indicate a major role of phlogopite in the mantle source.

Therefore, we suggest that the BAS could have originated from an enriched lithosphere mantle source in a garnet-stable area with abundant phlogopite and clinopyroxene. Recently, several Triassic intrusions have been identified in the Korean Peninsula, and comprise alkaline-feldspar granite and syenite with zircon and titanite $\mathrm{U}-\mathrm{Pb}$ ages of 234-210 Ma [56]. The BAS has similar Lu-Hf isotopic compositions and $\mathrm{U}-\mathrm{Pb}$ ages with syenite in the Korean Peninsula [56], indicating that they may have similar source area. 


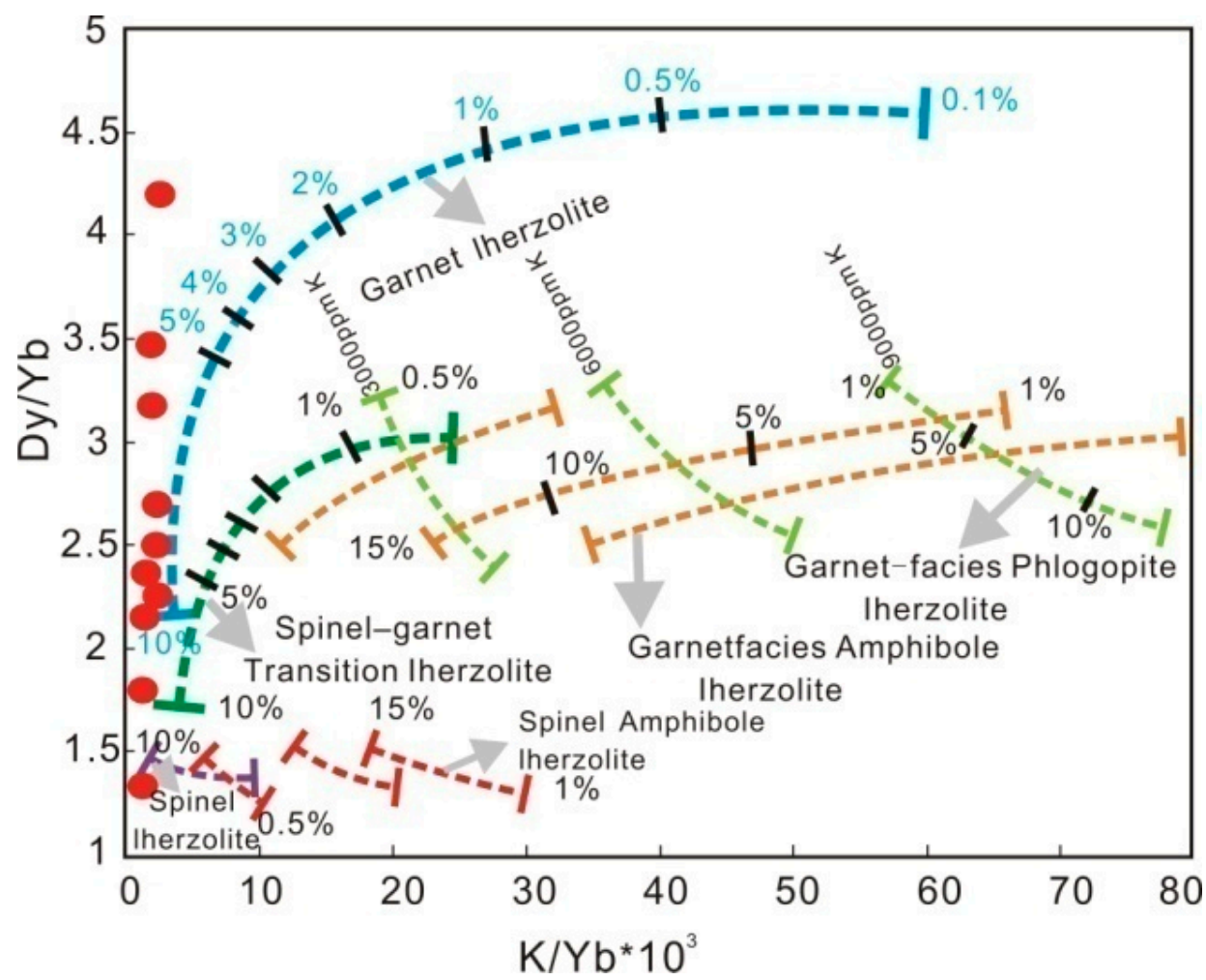

Figure 13. $\mathrm{K} / \mathrm{Yb}$ vs. Dy/Yb diagram for BAS. The samples plot suggested that garnet was a residual phase in the source(s) of the BAS. Percentages indicate degrees of partial melting (base drawing from [52].

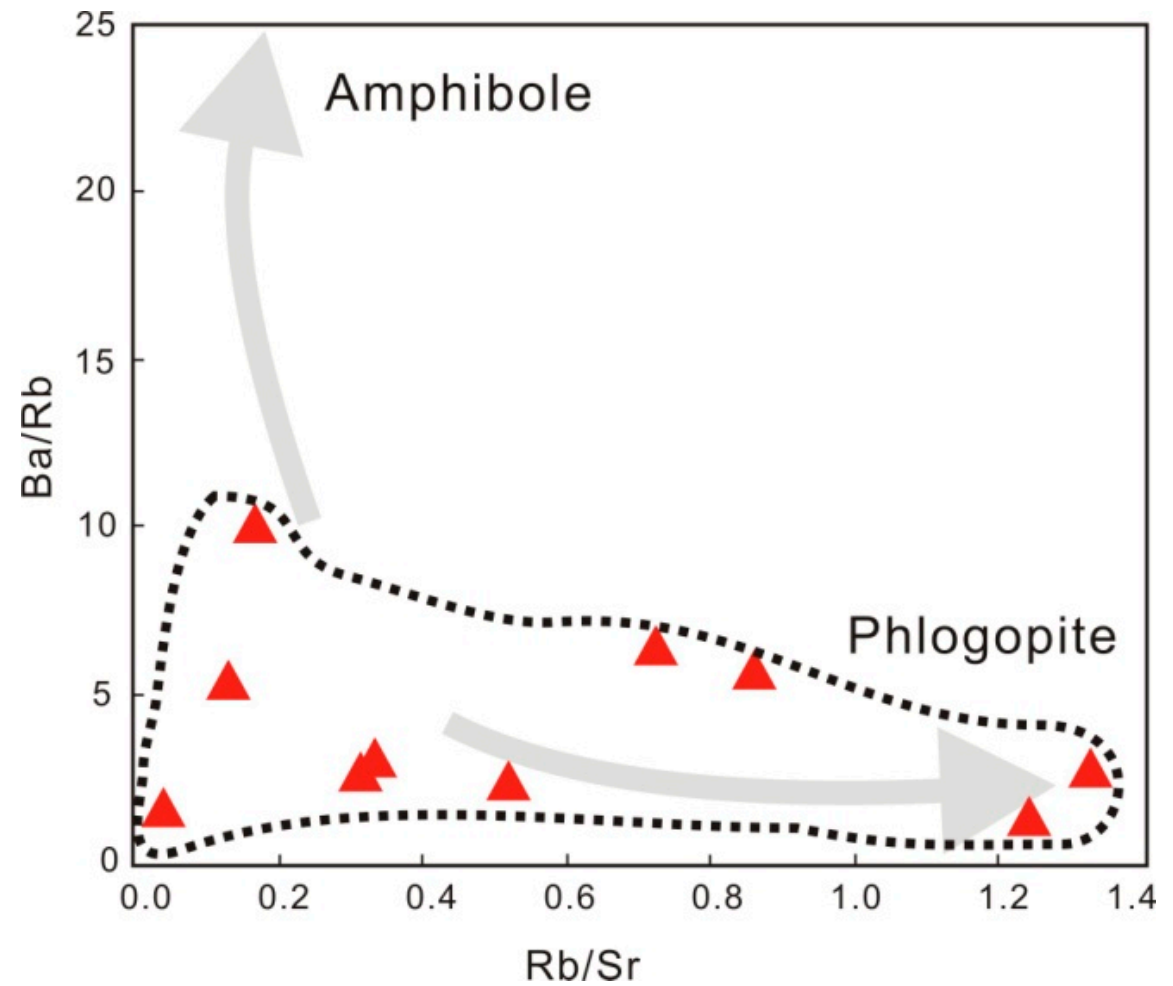

Figure 14. $\mathrm{Ba} / \mathrm{Rb}-\mathrm{Rb} / \mathrm{Sr}$ plot of syenite in Bailinchuan district. Low $\mathrm{Ba} / \mathrm{Rb}$ and high $\mathrm{Rb} / \mathrm{Sr}$ indicate a major role of phlogopite in the mantle source. 


\subsection{Tectonic Implications}

Alkaline rocks commonly occur in post-orogenic, rift or intraplate tectonic settings (e.g., $[8,10,12,13])$. They provide significant information on magmatic processes within the continental lithosphere and also contribute to the building of upper continental crust $[57,58]$. The Paleozoic to Mesozoic geodynamics of the northern NCC was mainly controlled by the subduction of the Paleo-Asian Ocean beneath the NCC and collision between the NCC and terrains of different types and derivation in the Paleo-Asian Ocean. Although the limited exposure of fragmentary ophiolite zones in the Central Asian orogenic belt have led to controversy concerning the final timing of amalgamation of these blocks, e.g., from the Late Silurian to Early Cretaceous (e.g., [59,60]), it has been accepted that the final closure time of the Paleo-Asian Ocean is Late Permian to Early Triassic [44]. This is supported by Late Paleozoic magmatism in the northern NCC [61]. The tectonic setting of Liaodong peninsula suggests that this region could be spatially under the influence of three major tectonic domains: (1) to the east is the Circle Pacific domain, subjected to Palaeo-Pacific plate subduction; (2) to the south is the Dabie-Sulu orogenic belt, associated with the subduction and subsequent collision between the Yangtze Craton and North China Craton; (3) to the north is the Central Asian orogenic belt, representing the closure of Palaeo-Asian Ocean and collision of an amalgamated North China-Mongolian plate with the Siberian plate. Temporally, the subduction of the Palaeo-Pacific plate occurred around the Early and Middle Jurassic [13]. Detailed zircon and monazite SHRIMP $\mathrm{U}-\mathrm{Pb}$ dating and inferred $P-T$ paths of metamorphic rocks documented that the continental collision and Dabie-Sulu ultrahigh pressure metamorphism (UHPM) took place at 220-240 Ma [62]. The final closure of the Palaeo-Asian Ocean and amalgamation between the Mongolian arc terranes and North China block is generally thought to have taken place around the Late Permian to Early Triassic [63,64]. Moreover, the collision at the eastern part might be later than the western part $[65,66]$. The Liaodong region might be under the influence of post-collisional extension of the Central Asian accretion belt in the Late Triassic. Sothe Palaeo-Asian tectonic system and the Dabie-Sulu orogenic belt were both under post-collisional extension in the Late Triassic when the magmatic activities took place.

As described above, the Late Triassic magmatic rocks are not isolated events in the Liaodong peninsula because contemporary igneous rocks were also documented in the Korea peninsula [66]. There is one long-extended Late Triassic post-collisional magmatic rocks belt (Table 4), of predominantly alkaline intrusions, developed along Liaodong and extending north into the Korea on the northeastern flank of the Sulu orogenic belt. For example, the Hekanzisyenites and Baotoudong syenites and BAS, Yaojiazhuang ultramafic-syenitic complex in the northern NCC and the Tokdal Complex in North Korea and Gwangcheon intrusive complex in South Korea. The Late Triassic alkaline rocks have similar geochemical characteristics in the Liaodong peninsula and the Korea peninsula, and are also interpreted to originate from an enriched mantle source with Yangtze crustal material $[66,67]$. The discrimination diagrams for tectonic settings of BAS suggest a post-collisional extension setting signature (Figure 15), which agrees with the fact that Central Asian orogenic belt had evolved into post-collisional extension with considerable asthenospheric mantle involvement at this time [7,66-68].

Based on some of the above some aspects, we propose that the Late Triassic magmatism in the Bailinchuan district is genetically associated with a post-collisional extension setting. Meanwhile, the Late Triassic BAS provide time constraints on the major geodynamic setting in the northern NCC. 

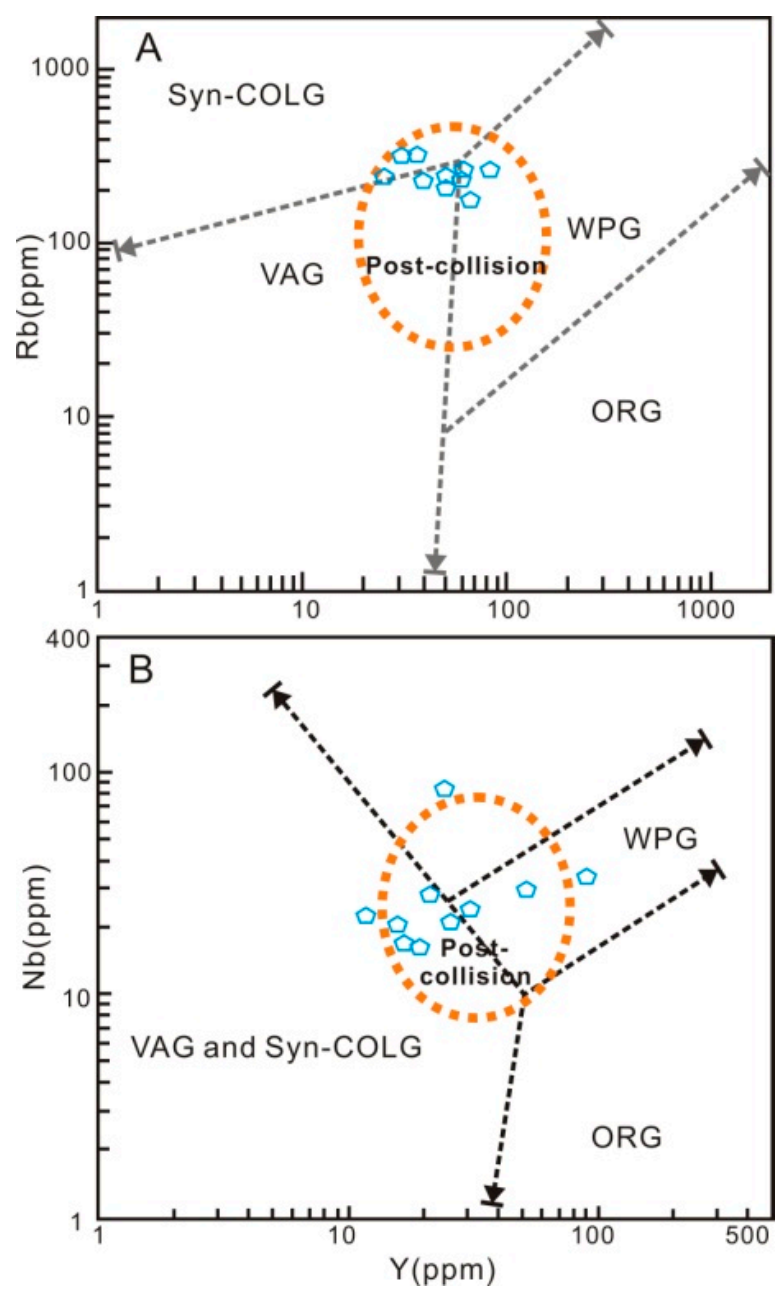

Figure 15. Discrimination diagrams for tectonic settings of BAS [67]. Abbreviations are VAG: volcanic arc granite; Syn-COLG: syn-collision granite; WPG: within-plate granite; ORG: oceanic ridge granite. Note: blue polygon represents syenite samples.

\section{Concluding Remarks}

Zircon U-Pb ages of BAS in the alkaline belt, northern North China Craton, indicate that these syenites were formed in the Late Triassic (226-229 Ma). Whole-rock geochemical and isotopic data reveal that the Late Triassic nepheline syenites were formed in a post-collisional extensional setting and were the result of crustal assimilation and fractional crystallization of a parental magma derived from a phlogopite-rich, enriched lithospheric mantle source in garnet-stable area via small degrees of partial melting. The Late Triassic Bailinchuan syenites provide time constraints on the major geodynamic setting in the northern NCC. The BAS was formed through fractional crystallization and accumulation of a $\mathrm{SiO}_{2}$-undersaturated potassic-ultrapotassic parental magma that is characterized by high contents of $\mathrm{K}_{2} \mathrm{O}, \mathrm{Na}_{2} \mathrm{O}$ and fluid $\left(\mathrm{H}_{2} \mathrm{O}\right)$, and by high temperature and high oxygen fugacity.

Author Contributions: X.C. wrote the paper and performed data treatment; J.X. and F.Y. formulated the problem, organized the research team, guided the study, participated in writing the manuscript and revised paper; H.W., G.Z. and H.Z. participated in experimental program.

Funding: This research was supported by the National Natural Science Foundation of China (41672070).

Acknowledgments: Thanks are due to three anonymous reviewers for their critical reviews and constructive comments, which attributed to the quality enhancement of this paper. We are also grateful to D.F Zhao and J.M Shi of SCCGS for providing help in field work.

Conflicts of Interest: The authors declare no conflict of interest. 


\section{References}

1. Lubala, R.T.; Frick, C.; Rogers, J.H.; Walraven, F. Petrogenesis of syenites and granites of the Schiel Alkaline Complex, Northern Transvaal, South Africa. J. Petrol. 1994, 102, 307-316. [CrossRef]

2. Wilson, M.; Downes, H.; Cebriá, J.M. Contrasting fractionation trends in coexisting continental alkaline magma series; Cantal, Massif Central, France. J. Petrol. 1995, 36, 1729-1753.

3. Yang, J.H.; Chung, S.L.; Wilde, S.A.; Wu, F.Y.; Chu, M.F.; Lo, C.H.; Fan, H.R. Petrogenesis of post-orogenic syenites in the Sulu Orogenic Belt, East China: Geochronological, geochemical and Nd-Sr isotopic evidence. Chem. Geol. 2005, 214, 99-125. [CrossRef]

4. Blichert-Toft, J.; Albarède, F. The Lu-Hf isotope geochemistry of chondrites and the evolution of the mantle-crust system. Earth Planet. Sci. Lett. 1997, 148, 243-258. [CrossRef]

5. Sutcliffe, R.H.; Smith, A.R.; Doherty, W.; Barnett, R.L. Mantle derivation of Archean amphibole-bearing granitoids and associated mafic rocks: Evidence from the southern Superior Province, Canada. Contrib. Mineral. Petrol. 1990, 105, 255-274. [CrossRef]

6. Lynch, D.J.; Musselman, T.E.; Gutmann, J.T.; Patchett, P.J. Isotopic evidence for the origin of Cenozoic volcanic rocks in the Pinacate volcanic field, northwestern Mexico. Lithos 1993, 29, 295-302. [CrossRef]

7. Jian, P.; Liu, D.Y.; Kroner, A.; Windley, B.F.; Shi, Y.R.; Zhang, W.; Zhang, F.Q.; Miao, L.C.; Zhang, L.Q.; Tomurhuu, D. Evolution of a Permian intraoceanic arc-trench system in the Solonker suture zone, Central Asian Orogenic Belt, China and Mongolia. Lithos 2010, 118, 169-190. [CrossRef]

8. Zhu, Y.S.; Yang, J.H.; Sun, J.F.; Zhang, J.H.; Wu, F.Y. Petrogenesis of coeval silica-saturated and silica-undersaturated alkaline rocks: Mineralogical and geochemical evidence from the Saima alkaline complex, NE China. J. Asian Earth Sci. 2016, 117, 184-207. [CrossRef]

9. Mu, B.L.; Yan, G.H. Geochemistry of Triassic alkaline or subalkaline igneous complexes in the Yan-Liao area and their significance. Acta Petrol. Sin. 1992, 66, 108-121. (In Chinese with English Abstract).

10. Yan, G.H.; Mu, B.L.; Xu, B.L. Triassic alkaline intrusives in the Yanliao-Yinshan area: Their chronology, Sr, $\mathrm{Nd}$ and $\mathrm{Pb}$ isotopic characteristics and their implications. Sci. China Ser. D 2000, 30, 383-387. [CrossRef]

11. Wu, F.Y.; Sun, D.Y.; Li, H.M. A-type granites in Northeastern China: Age and geochemical constraints on their petrogenesis. Chem. Geol. 2002, 187, 143-173. [CrossRef]

12. Wu, F.Y.; Yang, J.H.; Liu, X.M. Geochron-ological framework of the mesozoic granitic magmatism in the Liaodong Peninsula, North East China. Geol. J. China Univ. 2005, 11, 305-317. (In Chinese with English Abstract).

13. Jing, L.Z.; Guo, Y.J.; Ding, C.X. Geochromology and origin of Saima alkaline rocks in Liaoning province. Liaoning Geol 1995, 4, 257-271.

14. Tan, D.J.; Lin, J.Q.; Shan, X.L. On magma origin of Saima-Baiinchuan alkaline volcanic-intrusive complex. Geol. Rev 1999, 45, 474-481.

15. Hacker, B.R.; Ratschbacher, L.; Webb, L.; Ireland, T.; Walker, D.; Dong, S.W. U/Pb zircon ages constrain the architecture of the ultrahigh-pressure Qinling-Dabie Orogen, China. Earth Planet. Sci. Lett. 1998, 161, 215-230. [CrossRef]

16. Liu, F.L.; Xu, Z.Q.; Liou, J.G.; Song, B. SHRIMP U-Pb ages of ultrahigh-pressure and retrograde metamorphism of gneisses, south-western Sulu terrane, eastern China. J. Metamorph. Geol. 2004, 22, 315-326. [CrossRef]

17. Xu, W.L.; Zhou, Q.J.; Pei, F.P.; Yang, D.B.; Gao, S.; Li, Q.L.; Yang, Y.H. Destruction of the North China Craton: Delamination or thermal/chemical erosion? Mineral chemistry and oxygen isotope insights from websterite xenoliths. Gondwana Res. 2013, 23, 119-129. [CrossRef]

18. Yang, J.H.; Wu, F.Y.; Wilde, S.A.; Belousova, E.; Griffin, W.L. Mesozoic decratonization of the North China block. Geology 2008, 36, 467-470. [CrossRef]

19. Yang, J.H.; Sun, J.F.; Zhang, J.H.; Wilde, S.A. Petrogenesis of Late Triassic intrusive rocks in the northern Liaodong Peninsula related to decratonization of the North China Craton: Zircon U-Pb age and Hf-O isotope evidence. Lithos 2012, 153, 108-128. [CrossRef]

20. Xiao, W.J.; Windley, B.F.; Sun, S.; Li, J.L.; Huang, B.C.; Han, C.M.; Yuan, C.; Sun, M.; Chen, H.L. A Tale of Amalgamation of Three Permo-Triassic Collage Systems in Central Asia: Oroclines, Sutures, and Terminal Accretion. Annu. Rev. Earth Planet. Sci. 2015, 43, 477-507. [CrossRef] 
21. Lin, W.; Faure, M.; Monie, P. Mesozoic extensional tectonics in Eastern margin of Eurasia continent, the case study of South-Liaodong peninsula dome, NE China. J. Petrol. 2008, 116, 134-154.

22. Yang, J.H.; Wu, F.Y.; Zhang, Y.B. Identification of Meso-proterozoic zircons in a Triassic dolerite from the Liaodong Peninsula, East China. Chin. Sci. Bull. 2004, 49, 1958-1962. [CrossRef]

23. Chen, B.; Niu, X.L.; Wang, Z.Q.; Gao, L.; Wang, C. Geochronology, petrology, and geochemistry of the Yaojiazhuang ultramafic-syenitic complex from the North China Craton. Sci. China Earth Sci. 2013, 56, 1294-1307. [CrossRef]

24. Deng, J.F.; Su, S.G.; Mo, X.X.; Zhao, G.C.; Xiao, Q.H.; Ji, G.Y.; Qu, R.Z.; Zhao, H.L.; Luo, Z.H.; Wang, Y.; et al. The sequence of magmatic-tectonic events and orogenic processes of Yanshan belt, North China. Acta Geol. Sin. 2004, 78, 260-266. (In Chinese with English Abstract).

25. Chen, Z.B. Saima Alkaline Complex and Mineralization; Atomic Energy Press: Beijing, China, 1996; p. 305. (In Chinese with English Abstract).

26. Yuan, H.L.; Gao, S.; Dai, M.N.; Zong, C.L.; Gunther, D.; Fontaine, G.H.; Liu, X.M.; Diwu, C.R. Simultaneous determinations of $\mathrm{U}-\mathrm{Pb}$ age, $\mathrm{Hf}$ isotopes and trace element compositions of zircon by excimer laser-ablation quadrupole and multiple-collector ICP-MS. Chem. Geol. 2008, 247, 100-118. (In Chinese with English Abstract). [CrossRef]

27. Ludwig, K.R. ISOPLOT 3.0: A Geochronological Toolkit for Microsoft Excel. Berkeley Geochronol. Cent. Spec. Publ. 2003, 4, 1-71.

28. Bouvier, A.; Vervoort, J.D.; Patchett, P.J. The Lu-Hf and Sm-Nd isotopic compositionof CHUR: Constraints from unequilibrated chondrites and implications for the bulkcomposition of terrestrial planets. Earth Planet. Sci. Lett. 2008, 273, 48-57. [CrossRef]

29. Jahn, B.M.; Auvray, B.; Cornichet, J.; Bai, Y.L.; Shen, Q.H.; Liu, D.Y. 3.5 Ga old amphibolites from eastern Hebei Province, China: Field occurrence, petrography, Sm-Nd isochron age and REE geochemistry. Precambrian Res. 1987, 34, 311-346. [CrossRef]

30. Scherer, E.; Münker, C.; Mezger, K. Calibration of the lutetium-hafnium clock. Science 2001, 293, $683-687$. [CrossRef] [PubMed]

31. DePaolo, D.J. Neodymium isotpoes in the Colorado Front Range and crust-mantle evolution in the Proterozoic. Nature 1981, 291, 662-665. [CrossRef]

32. Eizenhöfer, P.R.; Zhao, G.C.; Zhang, J.; Han, Y.G.; Hou, W.Z.; Liu, D.X.; Wang, B. Geochemical characteristics of the Permian basins and their provenances across the Solonker Suture Zone: Assessment of net crustal growth during the closure of the Palaeo-Asian Ocean. Lithos 2015, 224-225, 240-255.

33. Taylor, S.R.; McLennan, S.M. The Continental Crust: Its Composition and Evolution; Blackwell: Oxford, UK, 1985; p. 312.

34. Baldwin, J.A.; Brown, M. Age and duration of ultrahigh-temperature metamorphism in the Anápolis-Itaucu Complex, Southern Brasília Belt, central Brazil-constraints from U-Pb geochronology, mineral rare earth element chemistry and trace-element thermometry. J. Metamorph. Geol. 2008, 26, 213-233. [CrossRef]

35. Degeling, H.S. Zr Equilibria in Metamorphic Rocks. Ph.D. Thesis, Australian National University, Canberra, Australia, Unpublished work. 2003; p. 231.

36. Troitzsch, U.; Ellis, D.J. The $\mathrm{ZrO}_{2}-\mathrm{TiO}_{2}$ phase diagram. J. Mater. Sci. 2005, 40, 4571-4577. [CrossRef]

37. Zack, T.; Moraes, R.; Kronz, A. Temperature dependence of $\mathrm{Zr}$ in rutile: Empirical calibration of a rutile thermometer. Contrib. Mineral. Petrol. 2004, 148, 471-488. [CrossRef]

38. Watson, E.B.; Wark, D.A.; Thomas, J.B. Crystallization thermometers for zircon and rutile. Contrib. Mineral. Petrol. 2006, 151, 413-433. [CrossRef]

39. Ferry, J.M.; Watson, E.B. New thermodynamic models and revised calibrations for the Ti-in-zircon and Zr-in-rutile thermometers. Contrib. Mineral. Petrol. 2007, 154, 429-437. [CrossRef]

40. Hayden, L.A.; Watson, E.B. Rutile saturation in hydrous siliceous melts and its bearing on Ti thermometry of quartz and zircon. Earth Planet. Sci. Lett. 2007, 258, 561-568. [CrossRef]

41. Xiao, W.J.; Windley, B.F.; Hao, J.; Zhai, M.G. Accretion leading to collision and the Permian Solonker suture, Inner Mongolia, China, termination of the central Asian orogenic belt. Tectonics 2003, 22, 1069. [CrossRef]

42. Windley, B.F.; Alexelev, D.; Xiao, W.J.; Kröner, A.; Badarch, G. Tectonic models for accretion of the Central Asian Orogenic Belt. J. Geol. Soc. 2007, 164, 31-47. [CrossRef] 
43. Ma, X.; Chen, B.; Chen, J.F.; Niu, X.L. Zircon SHRIMP U-Pb age, geochemical, Sr-Nd isotopic, and in-situ Hf isotopic data of the Late Carboniferous-Early Permian plutons in the northern margin of the North China Craton. Sci. China Earth Sci. 2013, 56, 126-144. [CrossRef]

44. Chen, B.; Jahn, B.M.; Wilde, S.; Xu, B. Two contrasting Paleozoic magmatic belts in northern Inner Mongolia, China: Petrogenesis and tectonic implications. Tectonophysics 2000, 328, 157-182. [CrossRef]

45. Eizenhöfer, P.R.; Zhao, G.C. Solonker Suture in East Asia and its bearing on the final closure of the eastern segment of the Palaeo-Asian Ocean. Earth Sci. Rev. 2017. [CrossRef]

46. Rudnick, R.L.; Gao, S.; Ling, W.L.; Liu, Y.S.; Mcdonough, W.F. Petrology and geochemistry of spinel peridotite xenoliths from Hannuoba and Qixia, North China Craton. Lithos 2004, 77, 609-637. [CrossRef]

47. O'Reilly, S.Y.; Griffin, W.L. Apatite in the mantle: Implications for metasomatic processes and high heat production in the Phanerozoic mantle. Lithos 2000, 53, 217-232. [CrossRef]

48. Gibson, S.A.; Thompson, E.N.; Leonardos, O.H.; Dickin, A.P.; Mitchell, J.G. The limited extent of plume-lithosphere interactions during continental flood-basalt genesis: Evidence from Cretaceous magmatism in southern Brazil. Contrib. Mineral. Petrol. 1999, 137, 147-169. [CrossRef]

49. Médard, E.; Schmidt, M.W.; Schiano, P.; Pttplini, L. Melting of amphibole-bearing wehrlites: An experimental study on the origin of ultra-calcic nepheline-normative melts. J. Petrol. 2006, 47, 481-504. [CrossRef]

50. Furman, T.; Graham, D. Erosion of lithospheric mantle beneath the East African Rift system: Geochemical evidence from the Kivu volcanic province. Lithos 1999, 48, 237-262. [CrossRef]

51. Spath, A.; LeRoex, A.P.; Duncan, R.A. The geochemistry of lavas from the Comores Archipelago, western Indian ocean: Petrogenesis and mantle source region characteristics. J. Petrol. 1996, 37, 961-991. [CrossRef]

52. Brenan, J.M.; Shaw, H.F.; Ryerson, F.J.; Phinney, D.L. Experimental determination of trace-element partitioning between pargasite and a synthetic hydrous and esitic melt. Earth Planet. Sci. Lett. 1995, 135, 1-11. [CrossRef]

53. Duggen, S.; Hoernle, K.; Bogaard, P.V.D.; Garbe-schönberg, D. Post-collisional transition from subduction to intraplate-type magmatism in the westernmost Mediterranean: Evidence for continental-edge delamination of subcontinental lithosphere. J. Petrol. 2005, 46, 1155-1201. [CrossRef]

54. Cho, D.L.; Lee, S.R.; Armstrong, R. Termination of the Permo-Triassic Songrim (Indosinian) orogeny in the Ogcheon belt, South Korea: Occurrence of ca. 220 Ma post-orogenic alkali granites and their tectonic implications. Lithos 2008, 105, 191-200. [CrossRef]

55. Turner, S.P.; Foden, J.D.; Morrison, R.S. Derivation of some A-type magmas by fractionation of basaltic magma; an example from the Padthaway Ridge, South Australia. Lithos 1992, 28, 151-179. [CrossRef]

56. Mingram, B.; Trubull, R.B.; Littman, S.; Gerstenberger, H. A petrogenetic study of anorogenic felsic magmatism in the Cretaceous Paresis ring complex, Namibia: Evidence for mixing of crust and mantle-derived components. Lithos 2000, 54, 1-22. [CrossRef]

57. Sengör, A.M.C.; Natal'in, B.A.; Burtman, V.S. Evolution of the Altaid tectonic collage and Palaeozoic crustal growth in Eurasia. Nature 1993, 364, 299-307. [CrossRef]

58. Williams, I.S.; Cho, D.L.; Kim, S.W. Geochronology, and geochemical and Nd-Sr isotopic characteristics of Triassic plutonic rocks in the Gyeonggi Massif, South Korea: Constraints on Triassic post-collisional magmatism. Lithos 2009, 107, 239-256. [CrossRef]

59. Nedelec, A.; Stephens, W.E.; Fallick, A.E. The Panafrican stratoid granites of Madagascar: Alkaline magmatism in a post-collisional extensional setting. J. Petrol. 1995, 36, 1367-1391. [CrossRef]

60. Zhao, Z.H.; Zhou, L.L. REE geochemistry of some alkaline rich intrusive rocks in China. Sci. China. Ser. D 1997, 40, 147-156. [CrossRef]

61. Wan, Y.S.; Li, R.W.; Wilde, S.A.; Liu, D.Y.; Chen, Z.Y.; Yan, L.; Song, T.R.; Yin, X.Y. UHP metamorphism and exhumation of the Dabie Orogen, China, evidence from SHRIMP dating of zircon and monazite from UHP granitic gneiss cobble from the Hefei Basin. Geochim. Cosmochim. Acta 2005, 69, 4333-4348. [CrossRef]

62. Xiao, W.J.; Windley, B.F.; Huang, B.C.; Han, C.M.; Yuan, C.; Chen, H.L.; Sun, M.; Sun, S.; Li, J.L. End-Permian to Mid-Triassic termination of the accretionary processes of the southern Altaids, implications for the geodynamic evolution, Phanerozoic continental growth, and metallogeny of Central Asia. Int. J. Earth Sci. 2009, 98, 1189-1217. [CrossRef]

63. Yang, J.H.; Wu, F.Y. Triassic magmatism and its relation to decratonization in the eastern North China Craton. Sci. China Ser. D Earth Sci. 2009, 52, 1319-1330. [CrossRef] 
64. Peng, P.; Zhai, M.G.; Guo, J.H. Petrogenesis of Triassic post-collisional syenite plutons in the Sino-Korean craton: An example from North Korea. Geol. Mag. 2008, 145, 637-647. [CrossRef]

65. Eizenhöfer, P.R.; Zhao, G.; Sun, M.; Zhang, J.; Han, Y.; Hou, W. Geochronologicaland Hf isotopic variability of detrital zircons in Paleozoic strata across the accretionarycollision zone between the North China craton and Mongolian arcs and tectonicimplications. Geol. Soc. Am. Bull. 2015, 127, 1422-1436. [CrossRef]

66. Eizenhöfer, P.R.; Zhao, G.; Zhang, J.; Sun, M. Final closure of the Paleo-Asian Oceanalong the Solonker Suture Zone: Constraints from geochronological and geochemicaldata of Permian volcanic and sedimentary rocks. Tectonics 2014, 33, 441-463. [CrossRef]

67. Pearce, J.A.; Harris, N.B.W.; Tindle, A.G. Trace element discriminationdiagrams for the tectonic interpretation of granitic rocks. J. Petrol. 1984, 25, 956-983. [CrossRef]

68. Ree, J.H.; Kwon, S.H.; Park, Y. Preteconic and posttectonic emplacements of the granitoids in the south central Okchon belt, South Korea: Implications for the timing of strike-slip shearing and thrusting. Tectonics 2001, 20, 850-867. [CrossRef]

(C) 2018 by the authors. Licensee MDPI, Basel, Switzerland. This article is an open access article distributed under the terms and conditions of the Creative Commons Attribution (CC BY) license (http://creativecommons.org/licenses/by/4.0/). 